\title{
UNIQUENESS FOR GIBBS MEASURES OF QUANTUM LATTICES IN SMALL MASS REGIME
}

\author{
Sergio ALBEVERIO $^{\text {a,b,c, d }}$, Yuri KONDRATIEV ${ }^{\text {a, b,e }}$, Yuri KOZITSKY ${ }^{\mathrm{f}}$, \\ Michael RÖCKNER ${ }^{\mathrm{g}}$ \\ ${ }^{a}$ Abteilung für Stochastik, Institut für Angewandte Mathematik, Universität Bonn, \\ 53115 Bonn, Germany \\ ${ }^{\mathrm{b}}$ Forschungszentrum BiBoS, Bielefeld, Germany \\ c SFB 237, Essen-Bochum-Düsseldorf, Germany \\ ${ }^{\mathrm{d}}$ CERFIM, Locarno, Switzerland \\ e Institute of Mathematics, Kiev, Ukraine \\ ${ }^{\mathrm{f}}$ Institute of Mathematics, Maria Curie-Sklodowska University, 20031 Lublin, Poland \\ g Fakultät für Mathematik, Universität Bielefeld, 33615 Bielefeld, Germany
}

Received 10 February 1999, revised 23 March 2000

ABSTRACT. - A model of interacting identical quantum particles performing one-dimensional anharmonic oscillations around their unstable equilibrium positions, which form the $d$-dimensional simple cubic lattice $\mathbb{Z}^{d}$, is considered. For this model it is proved that for every fixed value of the temperature $\beta^{-1}$ there exists a positive $m_{*}(\beta)$ such that for the values of the physical mass of the particle $m \in\left(0, m_{*}(\beta)\right)$, the set of tempered Gibbs measures consists of exactly one element. @ 2001 Éditions scientifiques et médicales Elsevier SAS

AMS classification: 60B05, 82B10

RÉSUMÉ. - On considère sur le réseau $\mathbb{Z}^{d}$ un modèle de particules quantiques en interaction soumises à oscillations unidimensionnelles anharmoniques autour de leur position d'équilibre instable. Pour ce modèle on montre que pour chaque valeur fixée de la température $\beta^{-1}$ il existe un réel $m_{*}(\beta)>0$ tel que pour les valeurs de la masse $m \in\left[0, m_{*}(\beta)\right]$, l'ensemble des état de Gibbs tempérés à un seul élément.@ 2001 Éditions scientifiques et médicales Elsevier SAS

\section{Introduction}

Let us first explain the problem studied in this paper from a purely probabilistic point of view. Subsequently, we shall describe the physics behind it.

We consider a lattice spin system over $\mathbb{Z}^{d}$ with single spin spaces equal to the space of continuous loops on $\mathbb{R}$ indexed by $[0, \beta]$. The single spin space is equipped with

E-mail addresses: albeverio@uni-bonn.de (S.Albeverio), kondratiev@uni-bonn.de (Y. Kondratiev), jkozi@golem.umcs.lublin.pl (Y. Kozitsky), roeckner@mathematik.uni-bielefeld.de (M. Röckner). 
the Brownian bridge measure multiplied by a density describing a polynomial selfinteraction. The interaction between the sites is attractive and of nearest neighbour type. Using the Dobrushin-Lanford-Ruelle formalism we define corresponding Gibbs measures through the associated local Gibbs specifications (see, e.g., [22]). In general, there may be infinitely many such Gibbs measures. However, one could expect that if the diffusion intensity is large enough, no phase transitions occur, i.e. there is a unique Gibbs measure. In the paper we give a complete proof of this fact based on Dobrushin's uniqueness criterion. The corresponding contraction condition is shown to be satisfied by using spectral properties of the diffusion generator and FKG (resp. GKS) inequalities. More details on this and the precise relation to previous papers [7-9] will be explained at the end of this section.

In the language of quantum statistical mechanics, the system we consider may be described as follows. To each point of the lattice $\mathbb{L}=\mathbb{Z}^{d}$ there is attached a quantum particle with the physical mass $m$ which has an unstable equilibrium position at this point. Such particles perform one-dimensional oscillations around their equilibrium positions and interact via an attractive potential. Similar systems have been studied for many years as quite realistic models of a crystalline substance undergoing structural phase transitions [17,35].

A full mathematical description of the equilibrium statistical mechanical properties of a quantum lattice system may be given by constructing its temperature Gibbs states. These are positive normalized functionals on von Neumann algebras whose elements (observables) represent physical quantities characterizing the system (see [16,25]). In the case of systems, for which the algebra of observables of every sub-system in a finite $\Lambda \subset \mathbb{L}$ may be realized as the $C^{*}$-algebra of bounded operators on a Hilbert space, the theory of temperature Gibbs states is well elaborated [16]. But if one needs to include into consideration also unbounded operators, as in the case considered in this paper, the situation becomes much more complicated and the construction of temperature Gibbs states even for the systems of non-interacting particles turns into a very hard task.

In 1975 a probabilistic approach to the construction of temperature Gibbs states has been initiated in [1]. It uses the integration theory on path spaces (see also $[2,11,12,14$, $23,25,34])$. In this approach the state, as a functional, of a sub-system in a finite $\Lambda$ at a temperature $T=\beta^{-1}$, is constructed by means of a probability measure $\mu_{\beta, \Lambda}$ defined on the space of continuous paths (loops). This measure is called local Euclidean Gibbs measure, and the infinite volume limits $\Lambda \nearrow \mathbb{L}$ of the sequences $\left\{\mu_{\beta, \Lambda}\right\}$, are exactly the Gibbs measures mentioned at the beginning of this introduction. Due to this fact, various probabilistic techniques became available for the description of equilibrium properties of quantum infinite-particle systems.

An alternative approach to the construction of temperature Gibbs states lies in the use of cluster expansions. We refer here to [6,29] where models with "light" particles were studied by means of different versions of this technique. In particular, in [6] the existence of temperature Gibbs states was shown by proving the convergence of cluster expansions at a fixed temperature and for small masses. In [29], for small masses, the convergence of the corresponding cluster expansions was proved for all values of the temperature. However, for systems with unbounded oscillations, as in the case considered in this work, the convergence of the cluster expansions, in itself, does not imply uniqueness. 
The most spectacular physical phenomenon in infinite-particle systems is a phase transition. It occurs when for the same values of the parameters describing a system, one has several temperature Gibbs states. In our case the phase transition is connected with the appearance of macroscopic displacements of particles from their equilibrium positions (a long-range order) if the dimension $d$, the mass $m$, the temperature $\beta^{-1}$, and the parameters of the potential energy satisfy certain conditions. A mathematical study of these phenomena was performed in various papers, see, e.g., [13,20,26,30]. The essential problem in this context is to understand the role of quantum effects in phase transitions in such models. By physical arguments (see [32] and Ch. 2.5.4.3 of [17]), the quantum effects may suppress the long-range ordering. For a model similar to the one considered in this work, this was proved in [36]. Later on it was shown in [3,4,27] that not only the long-range order but also any critical anomaly of the displacements of particles are suppressed if the model is "strongly quantum", which may occur in particular if the mass of the particle is small. Therefore, one may expect that the "strong quantumness" of the model implies the uniqueness of its temperature Gibbs states.

The same question may be considered in the above mentioned approach based on probabilistic methods. Namely, is it possible to prove uniqueness for the Euclidean Gibbs measures if the diffusion is intensive? So far, such uniqueness, for the model considered in this work, was proved to occur under conditions which are irrelevant to the diffusion intensity. We refer to [7-9] where this was done by means of logarithmic Sobolev inequalities. In this paper we present a proof for uniqueness of the Euclidean Gibbs measures for small values of the physical mass $m$ of the particle, which in probabilistic interpretation corresponds to large diffusion. We describe the diffusion intensity (see (3.34)) by a parameter, which is defined by the spectral properties of the diffusion generator $\widetilde{H}$ (i.e. the one-particle Hamiltonian) as $\mathcal{D}=m \widetilde{\Delta}^{2}$, where $\widetilde{\Delta}$ is the minimal distance between the eigenvalues of $\widetilde{H}$. We show (Lemma 3.4) that $\mathcal{D}$ tends to $+\infty$ when $m \searrow 0$. This enables us to obtain the contractivity which implies uniqueness. The research is performed by means of a version of the lattice approximation technique known in the Euclidean quantum field theory [33,34]. A similar approach has already been used in $[3,4,27]$. It makes possible to involve the physical mass into consideration, so that it appears explicitly in the uniqueness conditions.

The paper is organized as follows. In Section 2 we introduce temperature loop spaces and define Euclidean Gibbs measures. Section 3 contains the uniqueness theorem and its proof. It is based on Dobrushin's criterion, formulated in that section, and on a number of lemmas. Section 4 contains the proofs of these lemmas, which in turn are based on two correlation inequalities formulated here as Theorems 4.1 and 4.2. The proof of these theorems follows in Section 5.

\section{Temperature loop formalism for Gibbs measures}

We study Euclidean Gibbs measures on a temperature loop space describing equilibrium states at a temperature $\beta^{-1}$ of a system of identical quantum particles performing anharmonic one-dimensional oscillations around their unstable equilibrium positions, which form a lattice $\mathbb{L}=\mathbb{Z}^{d}, d \in \mathbb{N}$, (see $[1,23,25]$ for this approach). Consider 
the space of continuous periodic functions

$$
C\left(I_{\beta}\right) \stackrel{\text { def }}{=}\left\{\omega \in C\left(I_{\beta} \rightarrow \mathbb{R}\right) \mid \omega(0)=\omega(\beta)\right\}, I_{\beta}=[0, \beta],
$$

and let $\mathcal{H}_{\beta} \stackrel{\text { def }}{=} L^{2}\left(I_{\beta}\right)$ be the real Hilbert space of functions on $I_{\beta}$ which are square integrable with respect to the Lebesgue measure, equipped with inner product $(\cdot, \cdot)_{\beta}$ and norm $\|\cdot\|_{\beta}$. The temperature loop space is

$$
\Omega_{\beta} \stackrel{\text { def }}{=} C\left(I_{\beta}\right)^{\mathbb{L}}=\left\{\omega=\left(\omega_{l}\right)_{l \in \mathbb{L}} \mid \omega_{l} \in C\left(I_{\beta}\right)\right\} .
$$

For $\Lambda \subset \mathbb{L}$, we set

$$
\Omega_{\beta, \Lambda} \stackrel{\text { def }}{=}\left\{\omega_{\Lambda}=\left(\omega_{l}\right)_{l \in \Lambda} \mid \omega_{l} \in C\left(I_{\beta}\right)\right\} .
$$

The spaces $\Omega_{\beta, \Lambda}, \Omega_{\beta}$ are equipped with the product topology and with the $\sigma$-algebra $\mathcal{B}\left(\Omega_{\beta, \Lambda}\right)$ generated by the cylinder sets

$$
B_{\Delta} \stackrel{\text { def }}{=}\left\{\omega \mid \omega_{l} \in B_{l} \subset C\left(I_{\beta}\right), l \in \Delta, \Delta \in \mathcal{L}\right\},
$$

where $\mathcal{L}$ is the set of all finite subsets of $\mathbb{L}$ and $B_{l}$ are Borel subsets. Define on $\mathcal{H}_{\beta}$ the following strictly positive trace class operator

$$
S_{\beta}=\left(-m \Delta_{\beta}+1\right)^{-1},
$$

where $\Delta_{\beta}$ stands for the Laplace-Beltrami operator on $I_{\beta}$ (considered as a circle of length $\beta$ ). Let $\gamma_{\beta}$ be the Gaussian measure on $\mathcal{H}_{\beta}$, uniquely determined by its Fourier transform

$$
\int_{\mathcal{H}_{\beta}} \exp \left(\mathrm{i}(\varphi, \omega)_{\beta}\right) \gamma_{\beta}(d \omega)=\exp \left(-\frac{1}{2}\left(\varphi, S_{\beta} \varphi\right)_{\beta}\right), \quad \varphi \in \mathcal{H}_{\beta}
$$

For this measure, one may show that

$$
\int_{\mathcal{H}_{\beta}}\|\omega\|_{C\left(I_{\beta}\right)}^{M} \gamma_{\beta}(d \omega)<\infty, \quad \forall M \in \mathbb{N},
$$

and $\gamma_{\beta}\left(C\left(I_{\beta}\right)\right)=1$. This measure is canonically generated by the oscillator bridge process of length $\beta$ [34]. For a finite $\Lambda \subset \mathbb{L}$, we set

$$
\gamma_{\beta, \Lambda}\left(d \omega_{\Lambda}\right) \stackrel{\text { def }}{=} \bigotimes_{l \in \Lambda} \gamma_{\beta}\left(d \omega_{l}\right)
$$

For every $\zeta \in \Omega_{\beta}$ and each finite $\Lambda$, we define the Gibbs measure in $\Lambda$, subject to a chosen $\zeta$, as the following probability measure on $\Omega_{\beta, \Lambda}$

$$
\mu_{\beta, \Lambda}\left(d \omega_{\Lambda} \mid \zeta\right) \stackrel{\text { def }}{=} \Psi_{\beta, \Lambda}\left(\omega_{\Lambda} \mid \zeta\right) \gamma_{\beta, \Lambda}\left(d \omega_{\Lambda}\right)
$$




$$
\Psi_{\beta, \Lambda}\left(\omega_{\Lambda} \mid \zeta\right) \stackrel{\text { def }}{=} \frac{1}{Z_{\beta, \Lambda}(\zeta)} \exp \left\{-\Phi_{\beta, \Lambda}\left(\omega_{\Lambda} \mid \zeta\right)-\sum_{l \in \Lambda} \int_{I_{\beta}} V\left(\omega_{l}(\tau) d \tau\right\}\right.
$$

The polynomial

$$
V(x)=\sum_{s=2}^{p} b_{s} x^{2 s}-\frac{1}{2} a x^{2} \stackrel{\text { def }}{=} P_{p}(x)-\frac{1}{2} a x^{2}, \quad p \geqslant 2,
$$

where $a>1, b_{p}>0$, and $b_{s} \geqslant 0$ for all $s \leqslant p$, describes the self-interaction of the particles, whereas

$$
\begin{aligned}
\Phi_{\beta, \Lambda}\left(\omega_{\Lambda} \mid \zeta\right)= & \frac{J}{4} \int_{I_{\beta}}\left\{\sum_{l, l^{\prime} \in \Lambda} \varepsilon_{l l^{\prime}}\left(\omega_{l}(\tau)-\omega_{l^{\prime}}(\tau)\right)^{2}\right. \\
& \left.+2 \sum_{l \in \Lambda, l^{\prime} \in \Lambda^{c}} \varepsilon_{l l^{\prime}}\left(\omega_{l}(\tau)-\zeta_{l^{\prime}}(\tau)\right)^{2}\right\} d \tau,
\end{aligned}
$$

is the interaction of the particles in $\Lambda$ between themselves and with the particles outside $\Lambda$ fixed by the external boundary condition

$$
\zeta_{\Lambda^{c}} \in C\left(I_{\beta}\right)^{\Lambda^{c}}, \quad \Lambda^{c} \stackrel{\text { def }}{=} \mathbb{L} \backslash \Lambda
$$

Here $\varepsilon_{l l^{\prime}}=1$ for the pairs of nearest neighbors, i.e. if $\left|l-l^{\prime}\right|=1$, and is zero otherwise. $Z_{\beta, \Lambda}(\zeta)$ is the normalizing factor known also as the local partition function.

For $B \in \mathcal{B}\left(\Omega_{\beta}\right)$ and $\omega \in \Omega_{\beta}$, let $\mathbf{1}_{B}(\omega)$ take values 1, resp. 0, if $\omega$ belongs, resp. does not belong, to $B$. For a subset $\Lambda$ and a pair $\omega, \zeta \in \Omega_{\beta}$, let $\omega_{\Lambda} \times \zeta_{\Lambda^{c}}$ stand for $\xi \in \Omega_{\beta}$ such that $\xi_{l}=\omega_{l}$ if $l \in \Lambda$, and $\xi_{l}=\zeta_{l}$ for $l \in \Lambda^{c}$. Now we introduce a family of probability kernels $\left\{\pi_{\beta, \Lambda} \mid \Lambda \in \mathcal{L}\right\}$ between $\left(\Omega_{\beta}, \mathcal{B}\left(\Omega_{\beta}\right)\right)$ and $\left(\Omega_{\beta}, \mathcal{B}\left(\Omega_{\beta}\right)\right)$ by

$$
\pi_{\beta, \Lambda}(B \mid \zeta) \stackrel{\text { def }}{=} \int_{\Omega_{\beta, \Lambda}} \mathbf{1}_{B}\left(\omega_{\Lambda} \times \zeta_{\Lambda^{c}}\right) \mu_{\beta, \Lambda}\left(d \omega_{\Lambda} \mid \zeta\right) .
$$

These kernels satisfy the consistency condition [22]

$$
\int_{\Omega_{\beta}} \pi_{\beta, \Lambda}(d \omega \mid \zeta) \pi_{\beta, \Lambda^{\prime}}(B \mid \omega)=\pi_{\beta, \Lambda}(B \mid \zeta),
$$

which holds for arbitrary pairs $\Lambda^{\prime} \subset \Lambda$, each $B \in \mathcal{B}\left(\Omega_{\beta}\right)$, and $\zeta \in \Omega_{\beta}$.

DEFINITION 2.1. - The probability measure $\mu_{\beta}$ on $\left(\Omega_{\beta}, \mathcal{B}\left(\Omega_{\beta}\right)\right)$ is said to be a Euclidean Gibbs measure of the model considered at the temperature $\beta^{-1}$ if it satisfies the Dobrushin-Lanford-Ruelle (DLR) equilibrium equation

$$
\int_{\Omega_{\beta}} \mu_{\beta}(d \omega) \pi_{\beta, \Lambda}(B \mid \omega)=\mu_{\beta}(B)
$$

for all $\Lambda \in \mathcal{L}$ and $B \in \mathcal{B}\left(\Omega_{\beta}\right)$. 
Among all measures solving the DLR equation we distinguish a class of the so called tempered measures (see $[7,24])$. To this end we introduce

$$
\mathcal{S}^{\prime}(\mathbb{L}) \stackrel{\text { def }}{=} \text { ind } \lim _{p \in \mathbb{N}}\left\{u \in \mathbb{R}^{\mathbb{L}} \mid \sum_{l \in \mathbb{L}}(1+|l|)^{-p} u_{l}^{2}<\infty\right\} .
$$

Definition 2.2. - The class of tempered Gibbs measures $\mathcal{G}_{\beta}$ consists of the Euclidean Gibbs measures the moment sequences of which obey the condition

$$
\left(\left\langle\left\|\omega_{l}\right\|_{\beta}\right\rangle_{\mu_{\beta}}\right)_{l \in \mathbb{L}} \in \mathcal{S}^{\prime}(\mathbb{L}) .
$$

Here and further on we write

$$
\langle f\rangle_{\mu}=\int f d \mu
$$

if the integral makes sense.

As it has been proved in [10] (see also [7] and the references therein), the class $\mathcal{G}_{\beta}$ is actually nonempty. Moreover, the model has a critical point and long-range order behaviour if its parameters satisfy certain conditions (see, e.g., $[13,26,30])$. This means that for one and the same value of the parameters describing the model, $\mathcal{G}_{\beta}$ contains more than one element.

\section{Uniqueness theorem}

THEOREM 3.1. - Let $\mathcal{G}_{\beta}$ be given by Definition 2.2. Then for every $\beta$, there exists a positive $m_{*}(\beta)$ such that for all values of the mass $m \in\left(0, m_{*}(\beta)\right)$, the class $\mathcal{G}_{\beta}$ consists of exactly one element, that is $\left|\mathcal{G}_{\beta}\right|=1$.

The rest of the paper is devoted to the proof of this theorem.

Let $(\mathcal{X}, \rho)$ be a complete separable metric space, $\mathcal{M}$ be the set of all probability measures on $(\mathcal{X}, \mathcal{B}(\mathcal{X}))$, and

$$
\mathcal{M}_{1} \stackrel{\text { def }}{=}\left\{\mu \in \mathcal{M} \mid \int_{\mathcal{X}} \rho\left(y, y_{0}\right) \mu(d y)<\infty\right\},
$$

for some $y_{0} \in \mathcal{X}$. Let also $\operatorname{Lip}(\mathcal{X})$ stand for the set of Lipschitz functions $f: \mathcal{X} \rightarrow \mathbb{R}$, for which we write

$$
\begin{gathered}
{[f]_{\text {Lip }} \stackrel{\text { def }}{=} \sup \left\{\frac{|f(y)-f(z)|}{\rho(y, z)}: y, z \in \mathcal{X}, y \neq z\right\},} \\
\operatorname{Lip}_{1}(\mathcal{X}) \stackrel{\text { def }}{=}\left\{f \in \operatorname{Lip}(\mathcal{X}) \mid[f]_{\text {Lip }} \leqslant 1\right\} .
\end{gathered}
$$

Given $\mu_{1}, \mu_{2} \in \mathcal{M}_{1}$, we set

$$
R\left(\mu_{1}, \mu_{2}\right) \stackrel{\text { def }}{=} \sup \left\{\left|\int_{\mathcal{X}} f(y) \mu_{1}(d y)-\int_{\mathcal{X}} f(y) \mu_{2}(d y)\right|: f \in \operatorname{Lip}_{1}(\mathcal{X})\right\} .
$$


A key role in the proof will be played by Dobrushin's matrix. It is defined by the measures $\mu_{\beta, \Lambda}(2.7)-(2.10)$ with $\Lambda=\{l\}$. To simplify notations we set

$$
\xi_{\{l\}^{c}}=\xi_{l}^{c}, \quad \mu_{\beta,\{l\}}(\cdot \mid \xi)=\mu_{l}(\cdot \mid \xi) .
$$

Then the elements of Dobrushin's matrix are

$$
C_{l l^{\prime}}=\sup \left\{\frac{R\left(\mu_{l}(\cdot \mid \xi), \mu_{l}(\cdot \mid \eta)\right)}{\left\|\xi_{l^{\prime}}-\eta_{l^{\prime}}\right\|_{\beta}}: \xi, \eta \in \Omega_{\beta}, \xi_{l^{\prime}}^{c}=\eta_{l^{\prime}}^{c}, l, l^{\prime} \in \mathbb{L}\right\} .
$$

They will be used to check Dobrushin's criterion [18,19,22,28].

PROPOSITION 3.1 (Dobrushin's uniqueness criterion). - Let

$$
\sup \left\{\sum_{l \in \mathbb{L} \backslash\left\{l^{\prime}\right\}} C_{l l^{\prime}}: l \in \mathbb{L}\right\}<1,
$$

then there exists exactly one tempered Gibbs measure.

Directly from the definitions (2.7)-(2.10), (3.5) one obtains

$$
\begin{aligned}
\mu_{l}\left(d \omega_{l} \mid \xi\right)= & \frac{1}{Z_{l}(\xi)} \exp \left\{-d J\left\|\omega_{l}\right\|_{\beta}^{2}+J \sum_{l^{\prime}:\left|l-l^{\prime}\right|=1}\left(\omega_{l}, \xi_{l^{\prime}}\right)_{\beta}\right. \\
& \left.-\int_{I_{\beta}} V\left(\omega_{l}(\tau)\right) d \tau\right\} \gamma_{\beta}\left(d \omega_{l}\right) .
\end{aligned}
$$

This implies that $\mu_{l}\left(d \omega_{l} \mid \xi\right)=\mu_{l}\left(d \omega_{l} \mid \eta\right)$ if $\xi_{l^{\prime}}^{c}=\eta_{l^{\prime}}^{c}$ and $\left|l-l^{\prime}\right| \neq 1$. Hence

$$
R\left(\mu_{l}(\cdot \mid \xi), \mu_{l}(\cdot \mid \eta)\right)=0, \quad C_{l l^{\prime}}=0 \quad \text { for }\left|l-l^{\prime}\right| \neq 1 .
$$

If $\left|l-l^{\prime}\right|=1$, all $C_{l l^{\prime}}$ are equal to each other, thus the condition (3.7) is satisfied if:

$$
C_{l l^{\prime}} \stackrel{\text { def }}{=} C<\frac{1}{2 d}, \quad \text { for }\left|l-l^{\prime}\right|=1 .
$$

By the translation invariance of the model, each $\mu_{l}(\cdot \mid \xi)$ is a copy of the following measure

$$
\mu^{y}(d \omega)=\frac{1}{Z_{y}} \exp \left\{-J d\|\omega\|_{\beta}^{2}+J(\omega, y)_{\beta}-\int_{I_{\beta}} V(\omega(\tau)) d \tau\right\} \gamma_{\beta}(d \omega),
$$

where

$$
\mathcal{H}_{\beta} \ni y=\sum_{l^{\prime}:\left|l-l^{\prime}\right|=1} \xi_{l^{\prime}}
$$

and $Z_{y}$ is the normalizing constant. Thereafter, the Dobrushin coefficients (3.9) may be written as

$$
C=\sup \left\{\frac{R\left(\mu^{y}, \mu^{z}\right)}{\|x-y\|_{\beta}}: y, z \in \mathcal{H}_{\beta}, y \neq z\right\}
$$


Having in mind (3.4) let us estimate the variance of the function

$$
\mathcal{H}_{\beta} \ni y \mapsto\langle f\rangle_{\mu^{y}}=\int_{\mathcal{H}_{\beta}} f(\omega) \mu^{y}(d \omega) \in \mathbb{R}
$$

with a fixed $f \in \operatorname{Lip}_{1}\left(\mathcal{H}_{\beta}\right)$. This function is Fréchet differentiable [7] and its derivative in the direction $\varphi \in \mathcal{H}_{\beta}$ is

$$
\begin{aligned}
\left(\nabla_{y}\langle f\rangle_{\mu^{y}}, \varphi\right)_{\beta} & =J\left\{\left\langle f \cdot(\cdot, \varphi)_{\beta}\right\rangle_{\mu^{y}}-\langle f\rangle_{\mu^{y}}\left\langle(\cdot, \varphi)_{\beta}\right\rangle_{\mu^{y}}\right\} \\
& =J \operatorname{Cov}_{\mu^{y}}\left(f,(\cdot, \varphi)_{\beta}\right) .
\end{aligned}
$$

By the Schwarz inequality one has

$$
\left|\left(\nabla_{y}\langle f\rangle_{\mu^{y}}, \varphi\right)_{\beta}\right| \leqslant J \sqrt{\operatorname{Var}_{\mu^{y}} f} \cdot \sqrt{\operatorname{Var}_{\mu^{y}}(\cdot, \varphi)_{\beta}}
$$

where

$$
\begin{aligned}
& \operatorname{Var}_{\mu^{y}} f=\frac{1}{2} \int_{\mathcal{H}_{\beta}} \int_{\mathcal{H}_{\beta}}\left(f(\omega)-f\left(\omega^{\prime}\right)\right)^{2} \mu^{y}(d \omega) \mu^{y}\left(d \omega^{\prime}\right), \\
& \operatorname{Var}_{\mu^{y}}(\cdot, \varphi)_{\beta}=\frac{1}{2} \int_{\mathcal{H}_{\beta}} \int_{\mathcal{H}_{\beta}}\left(\omega-\omega^{\prime}, \varphi\right)_{\beta}^{2} \mu^{y}(d \omega) \mu^{y}\left(d \omega^{\prime}\right) .
\end{aligned}
$$

The idea of proving Theorem 3.1 may be outlined as follows. Suppose that we have estimated, uniformly for all $y \in \mathcal{H}_{\beta}$, the first variance by a continuous function of $\beta$, of the parameters $a, b_{s}(2.9)$, and of the mass $m$. Let also the second variance be bounded by a function of $\beta, a, b_{s}$, and $m$, multiplied by $\|\varphi\|_{\beta}^{2}$. Then the mean-value theorem together with (3.12) imply that the condition (3.9) is satisfied provided the product of the mentioned bounds is sufficiently small. Below we shall implement this idea.

One observes that (3.17) defines a quadratic form on $\mathcal{H}_{\beta}$

$$
\operatorname{Var}_{\mu^{y}}(\cdot, \varphi)_{\beta} \stackrel{\text { def }}{=}\left(\varphi, K_{y} \varphi\right)_{\beta},
$$

with the operator $K_{y}$ given as follows

$$
\left(K_{y} \varphi\right)(\tau)=\int_{I_{\beta}} K_{y}\left(\tau, \tau^{\prime}\right) \varphi\left(\tau^{\prime}\right) d \tau^{\prime}, \quad \tau \in I_{\beta}
$$

The kernel $K_{y}\left(\tau, \tau^{\prime}\right)$ is

$$
K_{y}\left(\tau, \tau^{\prime}\right)=\frac{1}{2} \int_{\mathcal{H}_{\beta}} \int_{\mathcal{H}_{\beta}}\left[\omega(\tau)-\omega^{\prime}(\tau)\right]\left[\omega\left(\tau^{\prime}\right)-\omega^{\prime}\left(\tau^{\prime}\right)\right] \mu^{y}(d \omega) \mu^{y}\left(d \omega^{\prime}\right) .
$$


One may show that it is a continuous function of $\tau, \tau^{\prime} \in I_{\beta}$. Clearly, for every $y \in \mathcal{H}_{\beta}$, the operator $K_{y}$ is symmetric and positive, and (cf. (2.5))

$$
\operatorname{trace}\left(K_{y}\right)=\frac{1}{2} \int_{\mathcal{H}_{\beta}} \int_{\mathcal{H}_{\beta}}\left\|\omega-\omega^{\prime}\right\|_{\beta}^{2} \mu^{y}(d \omega) \mu^{y}\left(d \omega^{\prime}\right)<\infty .
$$

For a bounded linear operator $A: \mathcal{H}_{\beta} \rightarrow \mathcal{H}_{\beta}$, let $\mathcal{S}_{p p}(A)$ denote the pure point spectrum and let $\|A\|$ stand for its operator norm. For a positive compact operator $A$, one has

$$
\|A\|=\max \mathcal{S}_{p p}(A) .
$$

On the other hand, if $A$ is symmetric and positive, then (see [31, p. 216])

$$
\|A\|=\sup \left\{\frac{(\varphi, A \varphi)_{\beta}}{\|\varphi\|_{\beta}^{2}}: \varphi \in \mathcal{H}_{\beta} \backslash\{0\}\right\} .
$$

Introduce

$$
\widetilde{V}(x)=\sum_{s=2}^{p} 2^{1-s} b_{s} x^{2 s}-\frac{1}{2} a x^{2}=\widetilde{P}_{p}(x)-\frac{1}{2} a x^{2},
$$

where $p, a, b_{s}$ are as in (2.9), and

$$
\widetilde{H}=-\frac{1}{2 m} \frac{d^{2}}{d x^{2}}+\frac{1}{2}(2 J d+1) q^{2}+\widetilde{V}(q) .
$$

The latter operator is defined in $L^{2}(\mathbb{R}, d x)$, the operator $q$ acts as follows

$$
(q \psi)(x)=x \psi(x) .
$$

Further, along with the measures defined by (2.7) and (3.10), we introduce

$$
\tilde{\mu}^{y}(d \omega)=\frac{1}{\widetilde{Z}_{y}} \exp \left\{-J d\|\omega\|_{\beta}^{2}+J(\omega, y)_{\beta}-\int_{I_{\beta}} \tilde{V}(\omega(\tau)) d \tau\right\} \gamma_{\beta}(d \omega),
$$

where $\widetilde{Z}_{y}$ is the normalizing constant.

It appears (see, e.g., [1,23]) that for a function $F: \mathcal{H}_{\beta} \rightarrow \mathbb{R}, F(\omega)=F_{1}(\omega(\tau)), \tau \in I_{\beta}$, which is integrable with respect to the measure $\tilde{\mu}^{y}$, one has

$$
\int_{\mathcal{H}_{\beta}} F(\omega) \tilde{\mu}^{y}(d \omega)=\frac{1}{\widetilde{Z}_{y}} \operatorname{trace}\left\{F_{1}(q) \exp [-\beta \widetilde{H}]\right\} .
$$

By means of $\tilde{\mu}^{y}$ we define by (3.19) the operator $\widetilde{K}_{y}$ with the kernel

$$
\widetilde{K}_{y}\left(\tau, \tau^{\prime}\right)=\frac{1}{2} \int_{\mathcal{H}_{\beta}} \int_{\mathcal{H}_{\beta}}\left[\omega(\tau)-\omega^{\prime}(\tau)\right]\left[\omega\left(\tau^{\prime}\right)-\omega^{\prime}\left(\tau^{\prime}\right)\right] \tilde{\mu}^{y}(d \omega) \tilde{\mu}^{y}\left(d \omega^{\prime}\right) .
$$


Its trace can be calculated as in (3.21) with the measure $\tilde{\mu}^{y}$ instead of $\mu^{y}$.

The construction of the bounds mentioned above is based upon the following lemmas, which are proved in Section 4.

LEMMA 3.1. - For every $y \in \mathcal{H}_{\beta}$ and all $\varphi \in \mathcal{H}_{\beta}$, one has

$$
\left(\varphi, K_{y} \varphi\right)_{\beta} \leqslant\left\|\widetilde{K}_{0}\right\|\|\varphi\|_{\beta}^{2}
$$

LEMMA 3.2. - For every $y \in \mathcal{H}_{\beta}$, one has

$$
\operatorname{trace}\left(K_{y}\right) \leqslant \operatorname{trace}\left(\widetilde{K}_{0}\right)
$$

It is known (see [15, p. 57]) that (the closure of) $\widetilde{H}$ is a self-adjoint operator with a discrete spectrum, all its eigenvalues $\widetilde{E}_{s}, s \in \mathbb{N}$, are simple. Set

$$
\widetilde{\Delta} \stackrel{\text { def }}{=} \inf \left\{\left|\widetilde{E}_{s}-\widetilde{E}_{s^{\prime}}\right|: s, s^{\prime} \in \mathbb{N}, s \neq s^{\prime}\right\} .
$$

LEMMA 3.3. - The following estimate holds

$$
\max \mathcal{S}_{p p}\left(\widetilde{K}_{0}\right) \leqslant \frac{1}{m \widetilde{\Delta}^{2}} .
$$

In what follows, as a parameter describing the diffusion intensity or, in quantum interpretation, the "quantumness" of the particle, one may choose

$$
\mathcal{D} \stackrel{\text { def }}{=} m \widetilde{\Delta}^{2}
$$

LEMMA 3.4. - There exists an independent of $m$ quantity $g_{0}>0$, such that for sufficiently small values of the mass $m$, the following estimate holds

$$
\frac{1}{m \tilde{\Delta}^{2}} \leqslant g_{0} m^{(p-1) /(p+1)},
$$

where $p$ is the same as in (2.9).

To estimate the variance (3.16) one may use the logarithmic Sobolev inequality, as it was done in [7].

LEMMA 3.5. - Let in (2.9) $p=2$, then for all $y \in \mathcal{H}_{\beta}$ and every $f \in \operatorname{Lip}_{1}\left(\mathcal{H}_{\beta}\right)$, one has

$$
\operatorname{Var}_{\mu^{y}} f \leqslant \frac{\mathrm{e}^{\beta \delta_{0}}}{2 J d+1+a / 4}, \quad \delta_{0}=\frac{25}{288} \frac{a^{2}}{b_{2}} .
$$

Another estimate of the variance of $f$ is linear in $\beta$. We will use it for $p>2$.

LEMMA 3.6. - There exists an independent of $m$ and $\beta$ quantity $h_{0}>0$ such that for all $y \in \mathcal{H}_{\beta}$, arbitrary $f \in \operatorname{Lip}_{1}\left(\mathcal{H}_{\beta}\right)$, the following estimate holds

$$
\operatorname{Var}_{\mu^{y}} f \leqslant \beta h_{0} m^{-1 /(p+1)} .
$$


Proof of Theorem 3.1. - First we estimate $\operatorname{Var}_{\mu^{y}}(\cdot, \varphi)_{\beta}$ given by (3.17), (3.18). By means of Lemma 3.1 and (3.22) one obtains

$$
\operatorname{Var}_{\mu^{y}}(\cdot, \varphi)_{\beta}=\left(\varphi, K_{y} \varphi\right)_{\beta} \leqslant\left\|\widetilde{K}_{0}\right\|\|\varphi\|_{\beta}^{2}=\max \mathcal{S}_{p p}\left(\widetilde{K}_{0}\right)\|\varphi\|_{\beta}^{2},
$$

and further by Lemmas 3.3, 3.4

$$
\operatorname{Var}_{\mu^{y}}(\cdot, \varphi)_{\beta} \leqslant g_{0} m^{(p-1) /(p+1)}\|\varphi\|_{\beta}^{2},
$$

that holds for sufficiently small $m$. For $p>2$, one may use (3.37) and choose $m$ so small that both latter estimates hold. This yields for the distance (3.4)

$$
R\left(\mu^{y}, \mu^{z}\right) \leqslant\|y-z\|_{\beta} J \sqrt{\beta \phi_{0} \chi_{0}} m^{\frac{p-2}{2(p+1)}} .
$$

Thus in view of (3.12), the condition of Dobrushin's criterion (3.9) is satisfied provided

$$
\beta m^{(p-2) /(p+1)}<\frac{1}{(2 J d)^{2} g_{0} h_{0}} .
$$

Therefore, in the case $p>2$ the upper bound for $m$ is

$$
m_{*}(\beta)=\frac{\beta^{-(p+1) /(p-2)}}{\left[(2 d J)^{2} g_{0} h_{0}\right]^{(p+1) /(p-2)}} .
$$

For $p=2$, we use (3.36) and obtain

$$
R\left(\mu^{y}, \mu^{z}\right) \leqslant\|y-z\|_{\beta} J \mathrm{e}^{\beta \delta_{0} / 2} m^{1 / 6} \sqrt{\frac{g_{0}}{2 J d+1+a / 4}},
$$

which implies in turn that Dobrushin's criterion is satisfied provided

$$
m<m_{*}(\beta) \stackrel{\text { def }}{=} \mathrm{e}^{-3 \beta \delta_{0}}\left(\frac{2 J d+1+a / 4}{(2 J d)^{2} g_{0}}\right)^{3} .
$$

\section{Proof of Lemmas}

The proof of Lemmas 3.1, 3.2 is based upon certain correlation inequalities, which we state just below and prove in the next section.

THEOREM 4.1 (FKG inequality). - For every finite $\Lambda$, arbitrary $\zeta \in \Omega_{\beta}$, all $l, l^{\prime} \in \Lambda$ and $\tau, \tau^{\prime} \in I_{\beta}$, one has

$$
\left\langle\omega_{l}(\tau) \omega_{l^{\prime}}\left(\tau^{\prime}\right)\right\rangle_{\mu_{\beta, \Lambda}(\cdot \mid \zeta)} \geqslant\left\langle\omega_{l}(\tau)\right\rangle_{\mu_{\beta, \Lambda}(\cdot \mid \zeta)}\left\langle\omega_{l^{\prime}}\left(\tau^{\prime}\right)\right\rangle_{\left.\mu_{\beta, \Lambda} \cdot \mid \zeta\right)},
$$

where the measure $\mu_{\beta, \Lambda}(\cdot \mid \zeta)$ is given by (2.7). In particular,

$$
K_{y}\left(\tau, \tau^{\prime}\right) \geqslant 0
$$

for all $y \in \mathcal{H}_{\beta}$ and $\tau, \tau^{\prime} \in I_{\beta}$. 
For a finite $\Lambda$, let us define the following probability measure on $\Omega_{\beta, \Lambda}$

$$
\varrho\left(d \varsigma_{\Lambda}\right)=\frac{1}{Y} \exp \left\{-\frac{J}{4} \sum_{l, l^{\prime} \in \Lambda} \varepsilon_{l l^{\prime}}\left\|\varsigma_{l}-\varsigma_{l^{\prime}}\right\|_{\beta}^{2}-\int_{I_{\beta}} \sum_{l \in \Lambda} W\left(\varsigma_{l}(\tau)\right) d \tau\right\} \gamma_{\beta, \Lambda}\left(d \varsigma_{\Lambda}\right),
$$

where $Y$ is the normalizing constant, the measure $\gamma_{\beta, \Lambda}$ is defined by (2.6), and the function $W: \mathbb{R} \rightarrow \mathbb{R}$ has the following form

$$
\begin{aligned}
& W(x)=w_{1} x^{2}+w_{2} x^{4}+\cdots+w_{p} x^{2 p}, \\
& w_{1} \in \mathbb{R}, w_{s} \geqslant 0, s=2, \ldots, p-1, w_{p}>0 .
\end{aligned}
$$

THEOREM 4.2 (GKS Inequality). - For a measure given by (4.3), the following inequality holds for all $s \in \mathbb{N}$, arbitrary $\lambda, l, l^{\prime} \in \Lambda$, and $t, \tau, \tau^{\prime} \in I_{\beta}$,

$$
\left\langle\left[\varsigma_{\lambda}(t)\right]^{2 s} \varsigma_{l}(\tau) \varsigma_{l^{\prime}}\left(\tau^{\prime}\right)\right\rangle_{\varrho} \geqslant\left\langle\left[\varsigma_{\lambda}(t)\right]^{2 s}\right\rangle_{\varrho}\left\langle\varsigma_{l}(\tau) \varsigma_{l^{\prime}}\left(\tau^{\prime}\right)\right\rangle_{\varrho} .
$$

Now we apply these inequalities to proving certain statements which then will be used to prove Lemmas 3.1, 3.2.

LEMma 4.1. - For every $y \in \mathcal{H}_{\beta}$ and all $\tau, \tau^{\prime} \in I_{\beta}$, one has

$$
K_{y}\left(\tau, \tau^{\prime}\right) \leqslant \widetilde{K}_{0}\left(\tau, \tau^{\prime}\right)
$$

Proof. - The representation (3.20) may be rewritten as

$$
K_{y}\left(\tau, \tau^{\prime}\right)=\iint_{\mathcal{H}_{\beta} \times \mathcal{H}_{\beta}} \frac{\omega(\tau)-\omega^{\prime}(\tau)}{\sqrt{2}} \cdot \frac{\omega\left(\tau^{\prime}\right)-\omega^{\prime}\left(\tau^{\prime}\right)}{\sqrt{2}}\left(\mu^{y} \otimes \mu^{y}\right)\left(d \omega, d \omega^{\prime}\right) .
$$

Here we apply the following orthogonal transformation of the space $\mathcal{H}_{\beta} \times \mathcal{H}_{\beta}$ :

$$
\begin{array}{ll}
\varsigma(\tau)=\left(\omega(\tau)-\omega^{\prime}(\tau)\right) / \sqrt{2}, & \omega(\tau)=(\varsigma(\tau)+v(\tau)) / \sqrt{2}, \\
v(\tau)=\left(\omega(\tau)+\omega^{\prime}(\tau)\right) / \sqrt{2}, & \omega^{\prime}(\tau)=(-\varsigma(\tau)+v(\tau)) / \sqrt{2},
\end{array}
$$

which yields (see (3.10), (2.9))

$$
\begin{aligned}
K_{y}\left(\tau, \tau^{\prime}\right)= & Z_{y}^{-2} \iint_{\mathcal{H}_{\beta} \times \mathcal{H}_{\beta}} \varsigma(\tau) \varsigma\left(\tau^{\prime}\right) \exp \left\{-\frac{1}{2}(2 J d-a)\left(\|\varsigma\|_{\beta}^{2}+\|v\|_{\beta}^{2}\right)\right. \\
& +\sqrt{2}(v, y)_{\beta}-\int_{I_{\beta}} \Pi(\varsigma(\tau) \mid v(\tau)) d \tau-\int_{I_{\beta}} \widetilde{P}_{p}(\varsigma(\tau)) d \tau \\
& \left.-\int_{I_{\beta}} \widetilde{P}_{p}(v(\tau)) d \tau\right\}\left(\gamma_{\beta} \otimes \gamma_{\beta}\right)(d \varsigma, d v)
\end{aligned}
$$

where we have put

$$
\Pi(x \mid u)=\sum_{s=1}^{p-1} \frac{x^{2 s}}{2 s !}\left(\frac{d^{2 s} \widetilde{P}_{p}}{d x^{2 s}}\right)(u) \stackrel{\text { def }}{=} \sum_{s=1}^{p-1} \pi_{s}(u) x^{2 s}, \quad x, u \in \mathbb{R} .
$$


For such $\Pi$ and the polynomials $P_{p}, \widetilde{P}_{p}$ given by (2.9), (3.24), one has

$$
P_{p}\left(\frac{x+u}{\sqrt{2}}\right)+P_{p}\left(\frac{x-u}{\sqrt{2}}\right)=\widetilde{P}_{p}(x)+\widetilde{P}_{p}(u)+\Pi(x \mid u) .
$$

Since all $b_{s}$ in (2.9), (3.24) are nonnegative, all the coefficients $\pi_{s}(u)$ are also nonnegative for all $u \in \mathbb{R}$. For $\vartheta \in[0,1]$, we set

$$
\Xi_{\vartheta}\left(\tau, \tau^{\prime}\right) \stackrel{\text { def }}{=}\left\langle\varsigma(\tau) \varsigma\left(\tau^{\prime}\right)\right\rangle_{\varrho_{\vartheta}},
$$

where expectation is taken with respect to the following probability measure on $\mathcal{H}_{\beta}$

$$
\begin{aligned}
\varrho_{\vartheta}(d \varsigma)= & \frac{1}{Y(\vartheta)} \exp \left\{-\frac{1}{2}(2 J d-a)\|\varsigma\|_{\beta}^{2}-\int_{I_{\beta}} \widetilde{P}_{p}(\varsigma(\tau)) d \tau\right. \\
& \left.-\vartheta \int_{I_{\beta}} \Pi(\varsigma(\tau) \mid v(\tau)) d \tau\right\} \gamma_{\beta}(d \varsigma), \\
Y(\vartheta)= & \int_{\mathcal{H}_{\beta}} \exp \left\{-\frac{1}{2}(2 J d-a)\|\varsigma\|_{\beta}^{2}-\int_{I_{\beta}} \widetilde{P}_{p}(\varsigma(\tau)) d \tau\right. \\
& \left.-\vartheta \int_{I_{\beta}} \Pi(\varsigma(\tau) \mid v(\tau)) d \tau\right\} \gamma_{\beta}(d \varsigma) .
\end{aligned}
$$

One observes that both $\Xi_{\vartheta}\left(\tau, \tau^{\prime}\right), Y(\vartheta)$ are continuous functions of $\vartheta \in[0,1]$. They are differentiable on $(0,1)$, and

$$
\begin{aligned}
\frac{\partial}{\partial \vartheta} \Xi_{\vartheta}\left(\tau, \tau^{\prime}\right)= & \frac{1}{Y(\vartheta)} \sum_{s=1}^{p-1} \int_{I_{\beta}} \pi_{s}(v(t))\left(\left\langle[\zeta(t)]^{2 s} \varsigma(\tau) \varsigma\left(\tau^{\prime}\right)\right\rangle_{\varrho_{\vartheta}}\right. \\
& \left.-\left\langle[\varsigma(t)]^{2 s}\right\rangle_{\varrho_{\vartheta}}\left\langle\varsigma(\tau) \varsigma\left(\tau^{\prime}\right)\right\rangle \varrho_{\vartheta}\right) d t .
\end{aligned}
$$

For every fixed $v \in \mathcal{H}_{\beta}, \tau \in I_{\beta}$, and $\vartheta \in[0,1]$, the coefficients $\pi_{s}(v(t)), s=1, \ldots, p-$ 1 , are nonnegative, hence this measure has the form (4.3) with a one-point subset $\Lambda$. Therefore, its moments possess the properties described by Theorem 4.2. The estimate (4.5) yields

$$
\frac{\partial}{\partial \vartheta} \Xi_{\vartheta}\left(\tau, \tau^{\prime}\right) \leqslant 0, \quad \forall \tau, \tau^{\prime} \in I_{\beta}
$$

which yields in turn

$$
0 \leqslant \Xi_{1}\left(\tau, \tau^{\prime}\right) \leqslant \Xi_{0}\left(\tau, \tau^{\prime}\right), \quad \forall \tau, \tau^{\prime} \in I_{\beta}, \forall v \in \mathcal{H}_{\beta} .
$$

The lower bound for $\Xi_{1}$ follows from (4.1). Applying these results in (4.9) we obtain

$$
K_{y}\left(\tau, \tau^{\prime}\right)=Z_{y}^{-2} \int_{\mathcal{H}_{\beta}} Y(1) \Xi_{1}\left(\tau, \tau^{\prime}\right) \exp \left\{-\frac{1}{2}(2 J d-a)\|v\|_{\beta}^{2}\right.
$$




$$
\begin{aligned}
& \left.+\sqrt{2}(v, y)_{\beta}-\int_{I_{\beta}} \widetilde{P}_{p}(v(t)) d t\right\} \gamma_{\beta}(d v) \\
& \leqslant \Xi_{0}\left(\tau, \tau^{\prime}\right) Z_{y}^{-2} \int_{\mathcal{H}_{\beta}} Y(1) \exp \left\{-\frac{1}{2}(2 J d-a)\|v\|_{\beta}^{2}+\sqrt{2}(v, y)_{\beta}\right. \\
& \left.-\int_{I_{\beta}} \widetilde{P}_{p}(v(t)) d t\right\} \gamma_{\beta}(d v)=\Xi_{0}\left(\tau, \tau^{\prime}\right)=\widetilde{K}_{0}\left(\tau, \tau^{\prime}\right) .
\end{aligned}
$$

Proof of Lemma 3.1. - Applying the inequalities (4.2), (4.6), and the representation of the norm of $\widetilde{K}_{0}(3.23)$, one gets

$$
\begin{aligned}
\left(\varphi, K_{y} \varphi\right)_{\beta} & =\left|\left(\varphi, K_{y} \varphi\right)_{\beta}\right| \leqslant \int_{I_{\beta}} \int_{I_{\beta}} K_{y}\left(\tau, \tau^{\prime}\right)|\varphi(\tau)|\left|\varphi\left(\tau^{\prime}\right)\right| d \tau d \tau^{\prime} \\
& \leqslant \int_{I_{\beta}} \int_{I_{\beta}} \widetilde{K}_{0}\left(\tau, \tau^{\prime}\right)\left|\varphi(\tau) \| \varphi\left(\tau^{\prime}\right)\right| d \tau d \tau^{\prime} \\
& \leqslant\left\|\widetilde{K}_{0}\right\|\|\varphi \mid\|_{\beta}^{2}=\left\|\widetilde{K}_{0}\right\|\|\varphi\|_{\beta}^{2} .
\end{aligned}
$$

Proof of Lemma 3.2. - One has

$$
\operatorname{trace}\left(K_{y}\right)=\int_{I_{\beta}} K_{y}(\tau, \tau) d \tau, \quad \operatorname{trace}\left(\widetilde{K}_{0}\right)=\int_{I_{\beta}} \widetilde{K}_{0}(\tau, \tau) d \tau,
$$

which gives (3.31) by means of (4.6).

Statements similar to Lemmas 3.3, 3.4 were proved in [4]. Here we give the proof of these lemmas to make the paper self-content.

Proof of Lemma 3.3. - For $y=0$, one has in (3.29)

$$
\widetilde{K}_{0}\left(\tau, \tau^{\prime}\right)=\int_{\mathcal{H}_{\beta}} \omega(\tau) \omega\left(\tau^{\prime}\right) \tilde{\mu}^{0}(d \omega)
$$

The periodicity of the loops $\omega(\tau)$ implies

$$
\widetilde{K}_{0}\left(\tau, \tau^{\prime}\right)=\widetilde{K}_{0}\left(\tau+\theta, \tau^{\prime}+\theta\right), \quad \forall \theta \in[0, \beta]
$$

where addition is modulo $\beta$. The latter yields in turn (see (3.28))

$$
\max \mathcal{S}_{p p}\left(\widetilde{K}_{0}\right)=\int_{I_{\beta}} \widetilde{K}_{0}(0, \tau) d \tau=\frac{1}{\widetilde{Z}} \int_{I_{\beta}} \operatorname{trace}\left\{q \mathrm{e}^{-\tau \widetilde{H}} q \mathrm{e}^{-(\beta-\tau) \widetilde{H}}\right\} d \tau
$$

where the operators $\widetilde{H}, q$ are defined by (3.25) and (3.26). Recall that the eigenvalues of $\widetilde{H}, \widetilde{E}_{s}$ are simple. Let $\psi_{s}$ stand for the corresponding eigenfunction and $q_{s s^{\prime}}=$ $\left(\psi_{s}, q \psi_{s^{\prime}}\right)_{\beta}$. For symmetry reasons $q_{s s}=0$, then 


$$
\max \mathcal{S}_{p p}\left(\widetilde{K}_{0}\right)=\frac{1}{\widetilde{Z}} \sum_{s, s^{\prime} \in \mathbb{N}, s \neq s^{\prime}}\left(q_{s s^{\prime}}\right)^{2} \frac{\left(\widetilde{E}_{s}-\widetilde{E}_{s^{\prime}}\right)\left(\mathrm{e}^{-\beta \widetilde{E}_{s^{\prime}}}-\mathrm{e}^{-\beta \widetilde{E}_{s}}\right)}{\left(\widetilde{E}_{s}-\widetilde{E}_{s^{\prime}}\right)^{2}} .
$$

The case of zero denominator is excluded, thus it may be estimated by means of (3.32), which yields

$$
\begin{aligned}
\max \mathcal{S}_{p p}\left(\widetilde{K}_{0}\right) & \leqslant \frac{1}{\widetilde{Z}} \frac{1}{\widetilde{\Delta}^{2}} \sum_{s, s^{\prime} \in \mathbb{N}}\left(q_{s s^{\prime}}\right)^{2}\left(\widetilde{E}_{s}-\widetilde{E}_{s^{\prime}}\right)\left(\mathrm{e}^{-\beta \widetilde{E}_{s^{\prime}}}-\mathrm{e}^{-\beta \widetilde{E}_{s}}\right) \\
& =\frac{1}{\widetilde{\Delta}^{2}} \cdot \frac{1}{\widetilde{Z}} \operatorname{trace}\left\{[q,[\widetilde{H}, q]] \mathrm{e}^{-\beta \widetilde{H}}\right\}=\frac{1}{m \widetilde{\Delta}^{2}},
\end{aligned}
$$

where $[\cdot, \cdot]$ stands for commutator.

Proof of Lemma 3.4. - For $\alpha>0$, consider the following unitary operator on $\mathcal{H}_{\beta}$

$$
\left(U_{\alpha} \psi\right)(x)=\alpha^{1 / 2} \psi(\alpha x) .
$$

Thus

$$
U_{\alpha}\left(\frac{d}{d x}\right) U_{\alpha}^{-1}=\alpha^{-1}\left(\frac{d}{d x}\right), \quad U_{\alpha} q U_{\alpha}^{-1}=\alpha q .
$$

Let $\alpha=m^{-1 /(2 p+2)}$. Then the operator

$$
\widetilde{H}(m)=m^{-p /(p+1)} T, \quad T \stackrel{\text { def }}{=} T_{0}+m^{1 /(p+1)} T_{1},
$$

is unitary equivalent to $\widetilde{H}$ given by $(3.25)$. Here

$$
\begin{gathered}
T_{0}=-\frac{1}{2} \frac{d^{2}}{d x^{2}}+2^{1-p} b_{p} q^{2 p}, \\
T_{1}=\frac{1}{2}(2 J d+1-a) m^{(p-2) /(p+1)} q^{2}+\sum_{l=2}^{p-1} 2^{1-l} m^{(p-l-1) /(p+1)} b_{l} q^{2 l} .
\end{gathered}
$$

Let $\Delta$ and $\Delta_{0}$ be defined by (3.32) but with the eigenvalues of the operators $T$ and $T_{0}$ respectively. Then

$$
\widetilde{\Delta}=m^{-p /(p+1)} \Delta .
$$

It can be observed that the operator $T$ is a perturbation of $T_{0}$, which is analytic (with respect to the variable $\left.\lambda=m^{1 /(p+1)}\right)$ at the point $\lambda=0$. Thus there exists a constant $c_{0}>0$ such that

$$
\Delta \geqslant c_{0} \Delta_{0}
$$

These arguments yield (3.35) with $g_{0}=1 /\left(c_{0} \Delta_{0}\right)^{2}$.

Proof of Lemma 3.5. - The estimate of the type of (3.36) has been proved in [7] by means of the logarithmic Sobolev inequality. Its realization (3.36) was obtained for the choice of $\widetilde{V}$ (3.24) with $p=2$. For more details we refer to Section 6 of [7]. 
Proof of Lemma 3.6. - For a Lipschitz function $f$, by means of (3.2), (3.3), (3.21), (3.28), (3.31), (4.17), (4.18) one obtains

$$
\begin{aligned}
\operatorname{Var}_{\mu^{y}} f & \leqslant \frac{1}{2} \int_{\mathcal{H}_{\beta}} \int_{\mathcal{H}_{\beta}}\left\|\omega-\omega^{\prime}\right\|_{\beta}^{2} \mu^{y}(d \omega) \mu^{y}\left(d \omega^{\prime}\right)=\operatorname{trace}\left(K_{y}\right) \\
& \leqslant \operatorname{trace}\left(\widetilde{K}_{0}\right)=\int_{I_{\beta}} \widetilde{K}_{0}(\tau, \tau) d \tau=\frac{\beta}{\widetilde{Z}} \operatorname{trace}\left(q^{2} \mathrm{e}^{-\beta \widetilde{H}}\right) \stackrel{\text { def }}{=} \beta\left\langle q^{2}\right\rangle .
\end{aligned}
$$

It turns out that $\max \mathcal{S}_{p p}\left(\widetilde{K}_{0}\right)$ may be expressed in terms of the Duhamel two-point function [21] and hence may be estimated from below as

$$
\beta\left\langle q^{2}\right\rangle f\left(\frac{\beta}{4 m\left\langle q^{2}\right\rangle}\right) \leqslant \max \mathcal{S}_{p p}\left(\widetilde{K}_{0}\right),
$$

where the function $f$ was introduced and estimated in [21]. It has the following bound

$$
\frac{1}{x}\left(1-\mathrm{e}^{-x}\right) \leqslant f(x)
$$

Applying in (4.25) this estimate together with (3.33), (4.22), (4.23), one gets

$$
2 m\left\langle q^{2}\right\rangle\left(1-\mathrm{e}^{-\beta / 4 m\left\langle q^{2}\right\rangle}\right) \leqslant 1 / \widetilde{\Delta} \leqslant m^{p /(p+1)} / c_{0} \Delta_{0} .
$$

Thus one may find a constant $h_{0}$ such that

$$
\left\langle q^{2}\right\rangle \leqslant h_{0} m^{-1 /(p+1)} .
$$

Applying this in (4.24) one obtains (3.37).

\section{Lattice approximation}

To prove Theorems 4.1 and 4.2 we will use a version of the lattice approximation technique known in the Euclidean quantum field theory [33,34]. Since our version has certain peculiarities, we give its detailed description.

For a $\zeta \in \Omega_{\beta}$, we define the measure

$$
\begin{aligned}
\varrho\left(d \omega_{\Lambda} \mid \zeta\right)= & \Upsilon\left(\omega_{\Lambda} \mid \zeta\right) \gamma_{\beta, \Lambda}\left(d \omega_{\Lambda}\right) \\
\stackrel{\text { def }}{=} & \frac{1}{Y(\zeta)} \exp \left\{-d J \sum_{l \in \Lambda}\left\|\omega_{l}\right\|_{\beta}^{2}+\frac{J}{2} \sum_{l, l^{\prime} \in \Lambda} \varepsilon_{l l^{\prime}}\left(\omega_{l}, \omega_{l^{\prime}}\right)_{\beta}\right. \\
& \left.+J \sum_{l \in \Lambda, l^{\prime} \in \Lambda^{c}} \varepsilon_{l l^{\prime}}\left(\omega_{l}, \zeta_{l^{\prime}}\right)_{\beta}-\int \sum_{I_{\beta}} W\left(\omega_{l}(\tau)\right) d \tau\right\} \gamma_{\beta, \Lambda}\left(d \omega_{\Lambda}\right),
\end{aligned}
$$

where $\gamma_{\beta, \Lambda}$ was given by (2.6), $Y(\zeta)$ is the normalization constant, and $W$ is given by (4.4). The measures (2.7), (4.3) may be written in this form. For $\tau_{1}, \ldots, \tau_{n} \in I_{\beta}$ and $l_{1}, \ldots, l_{n} \in \Lambda, \zeta \in \Omega_{\beta}$, let us consider 


$$
\begin{aligned}
\Gamma_{l_{1}, \ldots, l_{n}}\left(\tau_{1}, \ldots, \tau_{n}\right) & =\left\langle\omega_{l_{1}}\left(\tau_{1}\right), \ldots, \omega_{l_{n}}\left(\tau_{n}\right)\right\rangle_{\varrho(\cdot \mid \zeta)} \\
& =\int_{\Omega_{\beta, \Lambda}} \omega_{l_{1}}\left(\tau_{1}\right) \cdots \omega_{l_{n}}\left(\tau_{n}\right) \Upsilon\left(\omega_{\Lambda} \mid \zeta\right) \gamma_{\beta, \Lambda}\left(d \omega_{\Lambda}\right)
\end{aligned}
$$

Having (2.5) and employing the tightness of the measure $\gamma_{\beta, \Lambda}$, as well as the continuity of the loops $\omega_{\Lambda}$, one may prove the following statement.

Proposition 5.1. - For every $\zeta \in \Omega_{\beta, \Lambda}$ and all $l_{1}, \ldots, l_{n} \in \Lambda$, (5.2) is a continuous function of $\left(\tau_{1}, \ldots, \tau_{n}\right)$ on $I_{\beta}^{n}$.

The measure (2.6) is defined by the operator $S_{\beta}$ (2.3). The set of its eigenfunctions

$$
\begin{gathered}
\mathcal{E}=\left\{e_{k}(\tau) \mid k \in \mathcal{K}\right\}, \quad \mathcal{K}=\left\{k=\frac{2 \pi}{\beta} \kappa \mid \kappa \in \mathbb{Z}\right\}, \\
e_{k}(\tau)=\sqrt{\frac{2}{\beta}} \cos k \tau(k>0), \quad e_{k}(\tau)=-\sqrt{\frac{2}{\beta}} \sin k \tau(k<0), \quad e_{0}(\tau)=1 / \sqrt{\beta},
\end{gathered}
$$

may serve as a base of $\mathcal{H}_{\beta}$. Let $\left\{P_{k} \mid k \in \mathcal{K}\right\}$ be the family of orthogonal projectors onto the corresponding elements of $\mathcal{E}$. Then

$$
S_{\beta}=\sum_{k \in \mathcal{K}} \frac{1}{m k^{2}+1} P_{k}
$$

Now we choose $N=2 L, L \in \mathbb{N}$ and set

$$
\begin{aligned}
& \mathcal{K}_{N} \stackrel{\text { def }}{=}\left\{k=\frac{2 \pi}{\beta} \kappa \mid \kappa=-(L-1), \ldots, L\right\}, \\
& S_{\beta}^{(N)} \stackrel{\text { def }}{=} \sum_{k \in \mathcal{K}_{N}} \lambda_{k}^{(N)} P_{k}, \\
& \lambda_{k}^{(N)} \stackrel{\text { def }}{=} \frac{1}{m\left(\frac{2 N}{\beta}\right)^{2}\left[\sin \left(\frac{\beta}{2 N}\right) k\right]^{2}+1} .
\end{aligned}
$$

It is a technical exercise to prove the following statement.

Proposition 5.2. - The sequence of the finite-rank operators $\left\{S_{\beta}^{(N)}\right\}$ converges in the trace norm, when $N \rightarrow \infty$, to the operator $S_{\beta}$.

Let $\gamma_{\beta}^{(N)}$ be the symmetric Gaussian measure on $\mathcal{H}_{\beta}$ having $S_{\beta}^{(N)}$ as covariance operator. This measure may be written in a "coordinate form". To do this we introduce Gaussian measures on $\mathbb{R}, \chi_{k}^{(N)}, k \in \mathcal{K}$, such that

$$
\int_{\mathbb{R}} \exp (\mathrm{i} x u) \chi_{k}^{(N)}(d u)=\exp \left\{-\frac{1}{2} \lambda_{k}^{(N)} x^{2}\right\}
$$


where $\lambda_{k}^{(N)}$ are given by (5.7). Then

$$
\gamma_{\beta}^{(N)}(d \omega)=\bigotimes_{k \in \mathcal{K}_{N}} \chi_{k}^{(N)}(d \hat{\omega}(k)) \bigotimes_{k \in \mathcal{K} \backslash \mathcal{K}_{N}} \delta(\hat{\omega}(k)) d \hat{\omega}(k),
$$

where

$$
\omega(\tau)=\sum_{k \in \mathcal{K}} \hat{\omega}(k) e_{k}(\tau), \quad \hat{\omega}(k)=\int_{I_{\beta}} \omega(\tau) e_{k}(\tau) d \tau,
$$

and $\delta$ is the Dirac $\delta$-function on $\mathbb{R}$. Directly from the above statement one has

LEMMA 5.1. - The sequence $\left\{\gamma_{\beta}^{(N)}, N \in \mathbb{N}\right\}$ converges weakly, when $N \rightarrow \infty$, to the measure $\gamma_{\beta}$.

By means of $\left\{\gamma_{\beta}^{(N)}, N \in \mathbb{N}\right\}$ one may construct via (2.6), (2.7) corresponding approximations of the measure $\varrho(\cdot \mid \zeta)(5.1)$. The reason to use them is that by (5.9) the integrals with respect to the approximating measures may be written as integrals over finite-dimensional spaces. Then one could apply classical ferromagnetic interpretation, which would lead to the correlation inequalities we are going to prove.

It appears that we can get the ferromagnetic approximations of the function (5.2) only for the arguments belonging to $Q_{\beta}^{n} \subset I_{\beta}^{n}$, where $Q_{\beta}$ consists of such $\tau$ that $\tau / \beta$ is rational. Since $Q_{\beta}$ is dense in $I_{\beta}$, it is enough for our purposes in view of Proposition 5.1. In the sequel, we use the following types of functions $\Omega_{\beta, \Lambda} \rightarrow \mathbb{R}$ :

(i) $\omega_{\Lambda} \mapsto \omega_{l}(\tau), l \in \Lambda, \tau \in Q_{\beta}$;

(ii) $\omega_{\Lambda} \mapsto\left(\omega_{l}, \omega_{l^{\prime}}\right)_{\beta}, \omega_{\Lambda} \mapsto\left(\omega_{l}, \zeta_{l}\right)_{\beta}, l, l^{\prime} \in \Lambda, \zeta \in \Omega_{\beta}$;

(iii) $\omega_{\Lambda} \mapsto \int_{I_{\beta}} W\left(\omega_{l}(\tau)\right) d \tau$.

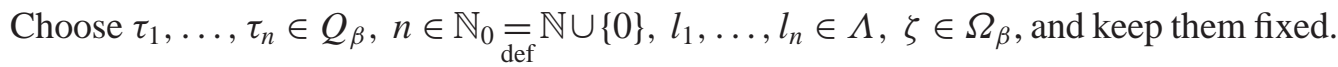
Then for $n \geqslant 1$, there exist tending to infinity sequences $\left\{N^{(k)}, k \in \mathbb{N}\right\},\left\{v_{j}^{(k)}, k \in \mathbb{N}\right\}$, $j=1, \ldots, n$, such that for all $k \in \mathbb{N}$,

$$
\tau_{j}=\frac{v_{j}^{(k)}}{N^{(k)}} \beta, \quad j=1, \ldots, n .
$$

Below we drop the symbol $(k)$ assuming that $N$ and $v_{j}$ tend to infinity in such a way that (5.12) holds. We also suppose that all $N$ are even. The set of $N$ satisfying (5.12) is denoted by $\mathcal{N}\left(\tau_{1}, \ldots, \tau_{n}\right)$. For $N \in \mathcal{N}\left(\tau_{1}, \ldots, \tau_{n}\right)$, we set

$$
\omega_{\Lambda}^{(N)}=\left(\omega_{l}^{(N)}\right)_{l \in \Lambda}, \quad \omega_{l}^{(N)} \stackrel{\text { def }}{=} \sum_{k \in \mathcal{K}_{N}} P_{k} \omega_{l} .
$$

For fixed $\tau_{1}, \ldots, \tau_{n} \in Q_{\beta}, l_{1}, \ldots, l_{n} \in \Lambda$, we write

$$
F\left(\omega_{\Lambda}\right)=\omega_{l_{1}}\left(\tau_{1}\right) \cdots \omega_{l_{n}}\left(\tau_{n}\right), \quad F^{(N)}\left(\omega_{\Lambda}\right) \stackrel{\text { def }}{=} F\left(\omega_{N}^{(N)}\right)
$$


Similarly

$$
\Upsilon^{(N)}\left(\omega_{\Lambda} \mid \zeta\right) \stackrel{\text { def }}{=} \Upsilon\left(\omega_{\Lambda}^{(N)} \mid \zeta\right)
$$

For a finite subset $\Lambda$ and $N \in \mathbb{N}$, we set

$$
\gamma_{\beta, \Lambda}^{(N)}\left(d \omega_{\Lambda}\right)=\bigotimes_{l \in \Lambda} \gamma_{\beta}^{(N)}\left(d \omega_{l}\right)
$$

The basic element of our construction is the following convergence statement, the proof of which will be given at the end of this section.

LEMMA 5.2. - For arbitrarily fixed $\tau_{1}, \ldots, \tau_{n} \in Q_{\beta}, l_{1}, \ldots, l_{n} \in \Lambda, \zeta \in \Omega_{\beta}$, the following convergence

$$
\begin{aligned}
\int_{\Omega_{\beta, \Lambda}} F\left(\omega_{\Lambda}\right) \Upsilon\left(\omega_{\Lambda} \mid \zeta\right) \gamma_{\beta, \Lambda}^{(N)}\left(d \omega_{\Lambda}\right) & =\int_{\Omega_{\beta, \Lambda}} F^{(N)}\left(\omega_{\Lambda}\right) \Upsilon^{(N)}\left(\omega_{\Lambda} \mid \zeta\right) \gamma_{\beta, \Lambda}^{(N)}\left(d \omega_{\Lambda}\right) \\
& \rightarrow \int_{\Omega_{\beta, \Lambda}} F\left(\omega_{\Lambda}\right) \Upsilon\left(\omega_{\Lambda} \mid \zeta\right) \gamma_{\beta, \Lambda}\left(d \omega_{\Lambda}\right),
\end{aligned}
$$

when $\mathcal{N}\left(\tau_{1}, \ldots, \tau_{n}\right) \ni N \rightarrow \infty$, holds.

Remark 5.1. - The above convergence does not follow automatically from Lemma 5.1 since the function under the integral in the left-hand side of (5.15) is unbounded on $\Omega_{\beta, \Lambda}$.

Having the representation (5.9), one may change the variables in

$$
\int_{\Omega_{\beta, \Lambda}} F^{(N)}\left(\omega_{\Lambda}\right) \Upsilon^{(N)}\left(\omega_{\Lambda} \mid \zeta\right) \gamma_{\beta, \Lambda}^{(N)}\left(d \omega_{\Lambda}\right)
$$

in such a way that in the new variables this integral would be finite-dimensional. To this end we pass to the variables $\hat{\omega}$ by means of the Fourier transformations (cf. (5.10))

$$
\omega_{l}(\tau)=\sum_{k \in \mathcal{K}} \hat{\omega}_{l}(k) e_{k}(\tau), \quad \hat{\omega}_{l}(k)=\int_{I_{\beta}} \omega_{l}(\tau) e_{k}(\tau) d \tau .
$$

Then for $Q_{\beta} \ni \tau=(\nu / N) \beta$, one has

$$
\omega_{l}^{(N)}(\tau)=\sum_{k \in \mathcal{K}_{N}} \hat{\omega}_{l}(k) e_{k}\left(\frac{v}{N} \beta\right)=\sqrt{\frac{N}{\beta}} \sum_{q \in \mathcal{Q}_{N}} \hat{\omega}_{l}\left(\frac{N}{\beta} q\right) \varepsilon_{q}(v),
$$

where

$$
\mathcal{Q}_{N} \stackrel{\text { def }}{=}\left\{q=\frac{2 \pi}{N} \kappa \mid \kappa=-(L-1), \ldots, L\right\}
$$


and for $v=0,1, \ldots, N-1,($ cf. $(5.3))$

$\varepsilon_{q}(v)=\sqrt{\frac{2}{N}} \cos q v(q>0) ; \quad \varepsilon_{q}(v)=-\sqrt{\frac{2}{N}} \sin q v(q<0) ; \quad \varepsilon_{0}(v)=\frac{1}{\sqrt{N}}$.

For the functions of the type (ii) taken at $\omega_{\Lambda}^{(N)}$, one has

$$
\left(\omega_{l}^{(N)}, \omega_{l^{\prime}}^{(N)}\right)_{\beta}=\sum_{k \in \mathcal{K}_{N}} \hat{\omega}_{l}(k) \hat{\omega}_{l^{\prime}}(k)=\sum_{q \in \mathcal{Q}_{N}} \hat{\omega}_{l}\left(\frac{N}{\beta} q\right) \hat{\omega}_{l^{\prime}}\left(\frac{N}{\beta} q\right)
$$

and

$$
\left(\omega_{l}^{(N)}, \zeta_{l}\right)_{\beta}=\sum_{k \in \mathcal{K}_{N}} \hat{\omega}_{l}(k) \hat{\zeta}_{l}(k), \quad \hat{\zeta}_{l}(k)=\int_{I_{\beta}} \zeta_{l}(\tau) e_{k}(\tau) d \tau .
$$

As for the functions of the type (iii), instead of (5.17) it is more convenient to use the following transformation of $\omega_{l}(\tau)$

$$
\omega_{l}(\tau)=\frac{1}{\sqrt{\beta}} \sum_{k \in \mathcal{K}} \tilde{\omega}_{l}(k) \exp (\mathrm{i} k \tau), \quad \tilde{\omega}_{l}(k)=\frac{1}{\sqrt{\beta}} \int_{I_{\beta}} \omega_{l}(\tau) \mathrm{e}^{-\mathrm{i} k \tau} d \tau .
$$

Then one has

$$
\int_{I_{\beta}} W\left(\omega_{l}^{(N)}(\tau)\right) d \tau=\sum_{s=1}^{p} w_{s} \int_{I_{\beta}}\left[\omega_{l}^{(N)}(\tau)\right]^{2 s} d \tau
$$

Further

$$
\begin{aligned}
\int_{I_{\beta}} & {\left[\omega_{l}^{(N)}(\tau)\right]^{2 s} d \tau } \\
& =\beta^{-s} \sum_{k_{1}, \ldots, k_{2 s} \in \mathcal{K}_{N}} \tilde{\omega}_{l}\left(k_{1}\right) \cdots \tilde{\omega}_{l}\left(k_{2 s}\right) \int_{I_{\beta}} \exp \left[\mathrm{i}\left(k_{1}+\cdots+k_{2 s}\right) \tau\right] d \tau \\
& =\beta^{-s+1} \sum_{k_{1}, \ldots, k_{2 s} \in \mathcal{K}_{N}} \tilde{\omega}_{l}\left(k_{1}\right) \cdots \tilde{\omega}_{l}\left(k_{2 s}\right) \delta\left(k_{1}+\cdots+k_{2 s}\right) .
\end{aligned}
$$

Here $\delta(0)=1, \delta(k)=0$ if $k \neq 0$. Having such representations, we introduce

$$
\begin{aligned}
& S_{l}(v)=\sqrt{\frac{\beta}{N}} \omega_{l}\left(\frac{v}{N} \beta\right), \quad l \in \Lambda, v=0,1, \ldots, N-1 ; \\
& \hat{S}_{l}(q)=\hat{\omega}_{l}\left(\frac{N}{\beta} q\right), \quad \tilde{S}_{l}(q)=\tilde{\omega}_{l}\left(\frac{N}{\beta} q\right), \quad q \in \mathcal{Q}_{N} ;
\end{aligned}
$$

for which one has

$$
S_{l}(v)=\sum_{q \in \mathcal{Q}_{N}} \hat{S}_{l}(q) \varepsilon_{q}(v)=\frac{1}{\sqrt{N}} \sum_{q \in \mathcal{Q}_{N}} \tilde{S}_{l}(q) \exp (\mathrm{i} q v)
$$




$$
\hat{S}_{l}(q)=\sum_{\nu=0}^{N-1} S_{l}(v) \varepsilon_{q}(v), \quad \tilde{S}_{l}(q)=\frac{1}{\sqrt{N}} \sum_{\nu=0}^{N-1} S_{l}(v) \exp (-\mathrm{i} q v) .
$$

Then (5.25) may be rewritten

$$
\begin{aligned}
\int_{I_{\beta}}\left[\omega_{l}^{(N)}(\tau)\right]^{2 s}= & \beta^{-s+1} \sum_{k_{1}, \ldots, k_{2 s} \in \mathcal{K}_{N}} \tilde{S}_{l}\left(\frac{\beta}{N} k_{1}\right) \cdots \tilde{S}_{l}\left(\frac{\beta}{N} k_{2 s}\right) \delta\left(k_{1}+\cdots+k_{2 s}\right) \\
= & \frac{1}{N^{s} \beta^{s-1}} \sum_{\nu_{1}, \ldots, \nu_{2 s}=0}^{N-1} S_{l}\left(v_{1}\right) \cdots S_{l}\left(v_{2 s}\right) \sum_{k_{1}, \ldots, k_{2 s} \in \mathcal{K}_{N}} \delta\left(k_{1}+\cdots+k_{2 s}\right) \\
& \times \exp \left\{-\frac{1 \beta}{N}\left(k_{1} v_{1}+\cdots+k_{2 s} v_{2 s}\right)\right\} \\
= & \frac{1}{N^{s} \beta^{s-1}} \sum_{v_{1}, \ldots, \nu_{2 s}=0}^{N-1} S_{l}\left(v_{1}\right) \cdots S_{l}\left(v_{2 s}\right) \\
& \times \sum_{\kappa_{1}, \ldots, \kappa_{2 s-1}=-L+1}^{L} \exp \left\{-\frac{2 \pi \mathrm{i}}{N} \kappa_{1}\left(v_{1}-v_{2 s}\right)\right\} \cdots \\
& \times \exp \left\{-\frac{2 \pi \mathrm{i}}{N} \kappa_{2 s-1}\left(v_{2 s-1}-v_{2 s}\right)\right\} \\
= & \frac{N^{2 s-1}}{N^{s} \beta^{s-1}} \sum_{\nu=0}^{N-1}\left[S_{l}(\nu)\right]^{2 s}=\frac{\beta}{N} \sum_{\nu=0}^{N-1}\left[\sqrt{\frac{N}{\beta}} S_{l}(v)\right]^{2 s} .
\end{aligned}
$$

Returning to (5.24) one obtains

$$
\int_{I_{\beta}} W\left(\omega_{l}^{(N)}(\tau)\right) d \tau=\frac{\beta}{N} \sum_{\nu=0}^{N-1} W\left(\sqrt{\frac{N}{\beta}} S_{l}(\nu)\right) .
$$

Accordingly,

$$
\begin{aligned}
\left(\omega_{l}^{(N)}, \omega_{l^{\prime}}^{(N)}\right)_{\beta} & =\sum_{q \in \mathcal{Q}_{N}} \hat{S}_{l}(q) \hat{S}_{l^{\prime}}(q)=\sum_{\nu=0}^{N-1} S_{l}(v) S_{l^{\prime}}(v), \\
\left(\omega_{l}^{(N)}, \zeta_{l}\right)_{\beta} & =\sum_{k \in \mathcal{K}_{N}} \hat{\omega}_{l}(k) \hat{\zeta}_{l}(k)=\sum_{\nu=0}^{N-1} S_{l}(v) X_{l}(v), \\
X_{l}(v) & \stackrel{\text { def }}{=} \sum_{q \in \mathcal{Q}_{N}} \hat{\zeta}_{l}\left(\frac{N}{\beta} q\right) \varepsilon_{q}(v) .
\end{aligned}
$$

At last, (5.18) takes the form

$$
\omega_{l_{j}}\left(\tau_{j}\right)=\omega_{l_{j}}\left(\frac{v_{j}}{N} \beta\right)=\sqrt{\frac{N}{\beta}} S_{l_{j}}\left(v_{j}\right), \quad j=1, \ldots, n .
$$

The next step is to construct the measure on a finite-dimensional space which has the mentioned ferromagnetic properties and such that (5.16) would be equal to the integral 
with respect to this measure. To this end by means of (5.9) we construct a finitedimensional analog of $\gamma_{\beta}^{(N)}$. First we introduce the following Gaussian measure on $\mathbb{R}^{N}$

$$
\sigma_{\beta}^{(N)}(d \hat{S})=\bigotimes_{q \in \mathcal{Q}_{N}} \tilde{\chi}_{q}^{(N)}(d \hat{S}(q))
$$

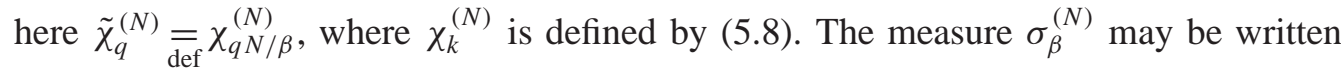
in the coordinates $\{S(v), v=0, \ldots, N-1\}$, connected to $\left\{\hat{S}(q), q \in \mathcal{Q}_{N}\right\}$ by the transformation (5.27), as follows

$$
\sigma_{\beta}^{(N)}(d S)=\frac{1}{C_{\beta, N}} \exp \left\{-\frac{m N^{2}}{2 \beta^{2}} \sum_{\nu=0}^{N-1}[S(v+1)-S(v)]^{2}-\frac{1}{2} \sum_{\nu=0}^{N-1}[S(v)]^{2}\right\} \bigotimes_{\nu=0}^{N-1} d S(v),
$$

with the convention $S(N)=S(0)$ and the normalizing constant $C_{\beta, N}$. Thus $\sigma_{\beta}^{(N)}$ may be considered as the Gibbs measure of a ferromagnetic chain of unbounded (Gaussian) spins (see, e.g., [33, p. 273] for more details). Now we set

$$
\begin{aligned}
\sigma_{\beta, \Lambda}^{(N)}\left(d S_{\Lambda}\right) \stackrel{\text { def }}{=} & \frac{1}{\left[C_{\beta, N}\right]^{|\Lambda|}} \exp \left\{-\frac{m N^{2}}{2 \beta^{2}} \sum_{l \in \Lambda} \sum_{\nu=0}^{N-1}\left[S_{l}(v+1)-S_{l}(v)\right]^{2}\right. \\
& \left.-\frac{1}{2} \sum_{l \in \Lambda} \sum_{\nu=0}^{N-1}\left[S_{l}(v)\right]^{2}\right\} \bigotimes_{l \in \Lambda}^{N-1} \bigotimes_{v=0} d S_{l}(v) .
\end{aligned}
$$

Here $S_{\Lambda}$ stands for the following $N|\Lambda|$-dimensional vector

$$
\left\{S_{l}(v), l \in \Lambda, v=0, \ldots, N-1\right\} .
$$

In what follows, by construction

$$
\begin{aligned}
& \int_{\Omega_{\beta, \Lambda}} F^{(N)}\left(\omega_{\Lambda}\right) \Upsilon^{(N)}\left(\omega_{\Lambda} \mid \zeta\right) \gamma_{\beta, \Lambda}^{(N)}\left(d \omega_{\Lambda}\right) \\
& =\frac{C_{N}(\zeta)}{Y(\zeta)}\left(\frac{N}{\beta}\right)^{n / 2} \int_{\mathbb{R}^{N|\Lambda|}} S_{l_{1}}\left(v_{1}\right) \cdots S_{l_{n}}\left(v_{n}\right) \rho_{\beta, \Lambda}^{(N)}\left(d S_{\Lambda} \mid X\right) \\
& =\frac{C_{N}(\zeta)}{Y(\zeta)}\left(\frac{N}{\beta}\right)^{n / 2}\left\langle S_{l_{1}}\left(v_{1}\right) \cdots S_{l_{n}}\left(v_{n}\right)\right\rangle_{\rho_{\beta, \Lambda}^{(N)}(\cdot \mid X)},
\end{aligned}
$$

where the probability measure $\rho_{\beta, \Lambda}^{(N)}(\cdot \mid X)$ is

$$
\begin{aligned}
\rho_{\beta, \Lambda}^{(N)}\left(d S_{\Lambda} \mid X\right) \stackrel{\text { def }}{=} & \frac{1}{C_{N}(\zeta)} \exp \left\{-d J \sum_{l \in \Lambda} \sum_{\nu=0}^{N-1}\left[S_{l}(v)\right]^{2}+\frac{J}{2} \sum_{l, l^{\prime} \in \Lambda} \varepsilon_{l l^{\prime}} \sum_{v=0}^{N-1} S_{l}(v) S_{l^{\prime}}(v)\right. \\
& +J \sum_{l \in \Lambda, l^{\prime} \in \Lambda^{c}} \varepsilon_{l l^{\prime}} \sum_{v=0}^{N-1} S_{l}(v) X_{l^{\prime}}(v) \\
& \left.-\frac{\beta}{N} \sum_{l \in \Lambda} \sum_{v=0}^{N-1} W\left(\sqrt{\frac{N}{\beta}} S_{l}(v)\right)\right\} \sigma_{\beta, \Lambda}^{(N)}\left(d S_{\Lambda}\right)
\end{aligned}
$$


Here $C_{N}(\zeta)$ is the normalization constant. Thus, Lemma 5.2 yields

$$
\begin{aligned}
& \Gamma_{l_{1}, \ldots, l_{n}}\left(\tau_{1}, \ldots, \tau_{n}\right) \\
& =\lim _{\mathcal{N}\left(\tau_{1}, \ldots, \tau_{n}\right) \ni N \rightarrow \infty} \frac{C_{N}(\zeta)}{Y(\zeta)}\left(\frac{N}{\beta}\right)^{n / 2}\left\langle S_{l_{1}}\left(v_{1}\right) \cdots S_{l_{n}}\left(v_{n}\right)\right\rangle_{\rho_{\beta, \Lambda}^{(N)}(\cdot \mid X)}, \\
& v_{j}=\frac{\tau_{j}}{\beta} N, j=1, \ldots, n .
\end{aligned}
$$

Proof of Theorems 4.1, 4.2. - Taking in (5.36) $n=0$ one gets that for all $\zeta \in \Omega_{\beta}$,

$$
C_{N}(\zeta) \rightarrow Y(\zeta), \quad N \rightarrow \infty
$$

The measure (5.35) corresponds to a general ferromagnet (see [33, p. 273]), for which the FKG inequality

$$
\frac{N}{\beta}\left\langle S_{l}(v) S_{l^{\prime}}\left(v^{\prime}\right)\right\rangle_{\left.\rho_{\beta, \Lambda}^{(N)} \cdot \mid X\right)} \geqslant \sqrt{\frac{N}{\beta}}\left\langle S_{l}(v)\right\rangle_{\varrho_{\beta, \Lambda}^{(N)}(\cdot \mid X)} \sqrt{\frac{N}{\beta}}\left\langle S_{l^{\prime}}\left(v^{\prime}\right)\right\rangle_{\varrho_{\beta, \Lambda}^{(N)}(\cdot \mid X)}
$$

holds (see Theorem VIII.16 of [33, p. 280]). Then it holds also for the limits, which means

$$
\left\langle\omega_{l}(\tau) \omega_{l^{\prime}}\left(\tau^{\prime}\right)\right\rangle_{\varrho(\cdot \mid \zeta)} \geqslant\left\langle\omega_{l}(\tau)\right\rangle_{\varrho(\cdot \mid \zeta)}\left\langle\omega_{l^{\prime}}\left(\tau^{\prime}\right)\right\rangle_{\varrho(\cdot \mid \zeta)} .
$$

Since the measure $\mu_{\beta, \Lambda}(\cdot \mid \zeta)$ given by (2.7)-(2.10) has the form (5.1), the above inequality may be rewritten as (4.1).

Now we take in (5.1) $\zeta=0$ and obtain $\varrho(\cdot \mid 0)=\varrho$, where the latter measure is given by (4.3). Thus to prove (4.5) we have to show that for $l_{1}=\cdots=l_{2 s}=\lambda, \tau_{1}=\cdots \tau_{2 s}=t$, the following inequality holds

$$
\Gamma_{l_{1}, \ldots, l_{2 s}, l, l^{\prime}}\left(\tau_{1}, \ldots, \tau_{2 s}, \tau, \tau^{\prime}\right) \geqslant \Gamma_{l_{1}, \ldots, l_{2 s}}\left(\tau_{1}, \ldots, \tau_{2 s}\right) \Gamma_{l, l^{\prime}}\left(\tau, \tau^{\prime}\right) .
$$

Taking into account (5.36), to this end we ought to have

$$
\left\langle\left[S_{\lambda}(v)\right]^{2 s} S_{l}\left(v_{1}\right) S_{l^{\prime}}\left(v_{2}\right)\right\rangle_{\rho_{\beta, \Lambda}^{(N)}(\cdot \mid 0)} \geqslant\left\langle\left[S_{\lambda}(v)\right]^{2 s}\right\rangle_{\rho_{\beta, \Lambda}^{(N)}(\cdot \mid 0)}\left\langle S_{l}\left(v_{1}\right) S_{l^{\prime}}\left(v_{2}\right)\right\rangle_{\rho_{\beta, \Lambda}^{(N)} \cdot(\cdot 0)},
$$

for $v=(t / \beta) N, v_{1}=(\tau / \beta) N, v_{2}=\left(\tau^{\prime} / \beta\right) N$. The measure $\rho_{\beta, \Lambda}^{(N)}(\cdot \mid 0)$ corresponds to an even ferromagnet, for which the GKS inequalities, in particular (5.37), hold (see Theorem VIII.14A in [33, p. 275]).

Proof of Lemma 5.2. - For arbitrary $\zeta \in \Omega_{\beta}$, the function $F^{(N)} \Upsilon^{(N)}(\cdot \mid \zeta)$ is bounded on $\Omega_{\beta, \Lambda}$. Thus, by Lemma 5.1 one has that for every fixed $N \in \mathcal{N}\left(\tau_{1}, \ldots, \tau_{n}\right)$,

$$
\begin{aligned}
& \int_{\Omega_{\beta, \Lambda}} F^{(N)}\left(\omega_{\Lambda}\right) \Upsilon^{(N)}\left(\omega_{\Lambda} \mid \zeta\right) \gamma_{\beta, \Lambda}^{(M)}\left(d \omega_{\Lambda}\right) \rightarrow \int_{\Omega_{\beta, \Lambda}} F^{(N)}\left(\omega_{\Lambda}\right) \Upsilon^{(N)}\left(\omega_{\Lambda} \mid \zeta\right) \gamma_{\beta, \Lambda}\left(d \omega_{\Lambda}\right), \\
& \quad \mathcal{N}\left(\tau_{1}, \ldots, \tau_{n}\right) \ni M \rightarrow \infty .
\end{aligned}
$$


Then the proof will be done if we show that for $N \rightarrow \infty$,

$$
\int_{\Omega_{\beta, \Lambda}} F^{(N)}\left(\omega_{\Lambda}\right) \Upsilon^{(N)}\left(\omega_{\Lambda} \mid \zeta\right) \gamma_{\beta, \Lambda}\left(d \omega_{\Lambda}\right) \rightarrow \int_{\Omega_{\beta, \Lambda}} F\left(\omega_{\Lambda}\right) \Upsilon\left(\omega_{\Lambda} \mid \zeta\right) \gamma_{\beta, \Lambda}\left(d \omega_{\Lambda}\right)
$$

One has

$$
\begin{aligned}
& \int_{\Omega_{\beta, \Lambda}}\left|F^{(N)}\left(\omega_{\Lambda}\right) \Upsilon^{(N)}\left(\omega_{\Lambda} \mid \zeta\right)-F\left(\omega_{\Lambda}\right) \Upsilon\left(\omega_{\Lambda} \mid \zeta\right)\right| \gamma_{\beta, \Lambda}\left(d \omega_{\Lambda}\right) \\
& \leqslant \int_{\Omega_{\beta, \Lambda}}\left|F^{(N)}\left(\omega_{\Lambda}\right)\right|\left|\Upsilon^{(N)}\left(\omega_{\Lambda} \mid \zeta\right)-\Upsilon\left(\omega_{\Lambda} \mid \zeta\right)\right| \gamma_{\beta, \Lambda}\left(d \omega_{\Lambda}\right) \\
& \quad+\int_{\Omega_{\beta, \Lambda}}\left|F^{(N)}\left(\omega_{\Lambda}\right)-F\left(\omega_{\Lambda}\right)\right| \Upsilon\left(\omega_{\Lambda} \mid \zeta\right) \gamma_{\beta, \Lambda}\left(d \omega_{\Lambda}\right) \\
& \stackrel{\text { def }}{=} I_{1}(N)+I_{2}(N) .
\end{aligned}
$$

Let us show that $I_{1}(N) \rightarrow 0$ when $N \rightarrow \infty$. In view of (2.5),

$$
\left|F^{(N)}\left(\omega_{\Lambda}\right)\right| \gamma_{\beta, \Lambda}\left(d \omega_{\Lambda}\right) \stackrel{\text { def }}{=} \phi\left(d \omega_{\Lambda}\right)
$$

is a finite positive measure on $\Omega_{\beta, \Lambda}$. Since it is tight, for every $\varepsilon>0$ and $C>0$, one may find a compact $\Omega_{\beta, \Lambda}^{\varepsilon} \subset \Omega_{\beta, \Lambda}$ such that $\phi\left(\Omega_{\beta, \Lambda} \backslash \Omega_{\beta, \Lambda}^{\varepsilon}\right)<(\varepsilon / 2 C)$. For all $N \in \mathcal{N}\left(\tau_{1}, \ldots, \tau_{n}\right)$ and $\zeta \in \Omega_{\beta}$, the function

$$
\left|\Upsilon^{(N)}\left(\omega_{\Lambda} \mid \zeta\right)-\Upsilon\left(\omega_{\Lambda} \mid \zeta\right)\right| \stackrel{\text { def }}{=} U_{N}\left(\omega_{\Lambda}\right)
$$

is continuous and bounded on $\Omega_{\beta, \Lambda}$ by an independent of $N$ constant $C(\zeta)$. The sequence $\left\{U_{N}\right\}$ converges point-wise to zero on $\Omega_{\beta, \Lambda}$. Thus, there exists a converging to zero sequence of positive numbers $\left\{u_{N}\right\}$, such that $U_{N}\left(\omega_{\Lambda}\right) \leqslant u_{N}$ uniformly on $\Omega_{\beta, \Lambda}^{\varepsilon}$. All these facts together imply

$$
I_{1}(N) \leqslant u_{N} \phi\left(\Omega_{\beta, \Lambda}^{\varepsilon}\right)+C(\zeta) \phi\left(\Omega_{\beta, \Lambda} \backslash \Omega_{\beta, \Lambda}^{\varepsilon}\right)<\varepsilon
$$

for sufficiently large $N$. Further

$$
\begin{aligned}
I_{2}(N)= & \int_{\Omega_{\beta, \Lambda}}\left|\omega_{l_{1}}\left(\tau_{1}\right) \cdots \omega_{l_{n}}\left(\tau_{n}\right)-\omega_{l_{1}}^{(N)}\left(\tau_{1}\right) \cdots \omega_{l_{n}}^{(N)}\left(\tau_{n}\right)\right| \Upsilon\left(\omega_{\Lambda} \mid \zeta\right) \gamma_{\beta, \Lambda}\left(d \omega_{\Lambda}\right) \\
\leqslant & \Upsilon_{\max }(\zeta) \sum_{i=1}^{n}\left\langle\left|\omega_{l_{1}}\left(\tau_{1}\right)\right| \cdots\left|\omega_{l_{i-1}}\left(\tau_{i-1}\right)\right|\left|\omega_{l_{i}}\left(\tau_{i}\right)-\omega_{l_{i}}^{(N)}\left(\tau_{i}\right)\right|\right. \\
& \left.\times\left|\omega_{l_{i+1}}^{(N)}\left(\tau_{i+1}\right)\right| \cdots\left|\omega_{l_{n}}^{(N)}\left(\tau_{n}\right)\right|\right\rangle_{\gamma_{\beta, \Lambda}} \\
\leqslant & \Upsilon_{\max }(\zeta) \sum_{i=1}^{n}\left\{\left\langle\left|\omega_{l_{i}}\left(\tau_{i}\right)-\omega_{l_{i}}^{(N)}\left(\tau_{i}\right)\right|^{2}\right\rangle_{\gamma_{\beta, \Lambda}}\right\}^{1 / 2}\left\{\left\langle\left|\omega_{l_{1}}\left(\tau_{1}\right)\right|^{2} \cdots\left|\omega_{l_{i-1}}\left(\tau_{i-1}\right)\right|^{2}\right.\right.
\end{aligned}
$$


S. ALBEVERIO ET AL. / Ann. Inst. H. Poincaré, Probab. Statist. 37 (2001) 43-69

$$
\begin{gathered}
\left.\left.\times\left|\omega_{l_{i+1}}^{(N)}\left(\tau_{i+1}\right)\right|^{2} \cdots\left|\omega_{l_{n}}^{(N)}\left(\tau_{n}\right)\right|^{2}\right\rangle_{\gamma_{\beta, \Lambda}}\right\}^{1 / 2} \\
\leqslant n C_{n} \Upsilon_{\max }(\zeta) \sup _{l \in \Lambda} \sup _{\tau \in I_{\beta}}\left\{\left\langle\left[\omega_{l}(\tau)-\omega_{l}^{(N)}(\tau)\right]^{2}\right\rangle_{\gamma_{\beta, \Lambda}}\right\}^{1 / 2} .
\end{gathered}
$$

Here $C_{n}$ is an independent of $N$ upper bound of the moments of $\omega_{l}$ and $\omega_{l}^{(N)}$, which exists in view of (2.5), and

$$
\Upsilon_{\max }(\zeta) \stackrel{\text { def }}{=} \sup _{\omega_{\Lambda} \in \Omega_{\beta, \Lambda}} \Upsilon\left(\omega_{\Lambda} \mid \zeta\right)
$$

In view of (5.13), (5.23) one may write

$$
\begin{aligned}
& \left\langle\left[\omega_{l}(\tau)-\omega_{l}^{(N)}(\tau)\right]^{2}\right\rangle_{\gamma_{\beta, \Lambda}} \\
& \quad=\frac{1}{\beta} \sum_{k, k^{\prime} \in \mathcal{K}_{N}^{c}}\left\langle\tilde{\omega}_{l}(k) \tilde{\omega}_{l}\left(-k^{\prime}\right)\right\rangle_{\gamma_{\beta, \Lambda}} \exp \left[\mathrm{i}\left(k-k^{\prime}\right) \tau\right], \quad \mathcal{K}_{N}^{c}=\mathcal{K} \backslash \mathcal{K}_{N} .
\end{aligned}
$$

Applying again (5.23) one obtains

$$
\left\langle\left[\omega_{l}(\tau)-\omega_{l}^{(N)}(\tau)\right]^{2}\right\rangle_{\gamma_{\beta}, \Lambda}=\frac{2}{\beta} \sum_{k \in \mathcal{K}_{N}^{c}} \int_{I_{\beta}} \Gamma_{l, l}^{(0)}(0, t) \cos (k t) d t,
$$

where $\Gamma^{(0)}$ is defined by (5.2) but with $\gamma_{\beta, \Lambda}$ instead of $\varrho(\cdot \mid \zeta)$. Define on $L^{2}\left(\mathbb{R}^{\Lambda}, d x_{\Lambda}\right)$ the following operator (cf. (3.25), (3.26))

$$
H^{(0)} \stackrel{\text { def }}{=} \sum_{l \in \Lambda}\left(-\frac{1}{2 m} \frac{d^{2}}{d x_{l}^{2}}+\frac{1}{2} q_{l}^{2}\right) \text {. }
$$

Let also $\left\{E_{s}^{(0)}, s \in \mathbb{N}\right\},\left\{\psi_{s}^{(0)}, s \in \mathbb{N}\right\}$ stand for its eigenvalues and eigenfunctions respectively. Denote $\left(q_{l}\right)_{s s^{\prime}}=\left(\psi_{s}^{(0)}, q_{l} \psi_{s^{\prime}}^{(0)}\right)_{L^{2}\left(\mathbb{R}^{\Lambda}, d x_{\Lambda}\right)}$. For $k \neq 0$, similarly to (4.19) one obtains

$$
\begin{aligned}
\int_{I_{\beta}} \Gamma_{l, l}^{(0)}(0, t) \cos (k t) d t & =\frac{1}{Z^{(0)}} \int_{I_{\beta}} \operatorname{trace}\left\{q_{l} \mathrm{e}^{-t H^{(0)}} q_{l} \mathrm{e}^{-(\beta-t) H^{(0)}}\right\} \cos (k t) d t \\
& =\frac{1}{Z^{(0)}} \sum_{s, s^{\prime} \in \mathbb{N}}\left(q_{l}\right)_{s s^{\prime}}^{2} \frac{\left(E_{s}^{(0)}-E_{s^{\prime}}^{(0)}\right)\left(\mathrm{e}^{-\beta E_{s^{\prime}}^{(0)}}-\mathrm{e}^{-\beta E_{s}^{(0)}}\right)}{\left(E_{s}^{(0)}-E_{s^{\prime}}^{(0)}\right)^{2}+k^{2}} \\
& \leqslant \frac{1}{k^{2}} \cdot \frac{1}{Z^{(0)}} \sum_{s, s^{\prime} \in \mathbb{N}}\left(q_{l}\right)_{s s^{\prime}}^{2}\left(E_{s}^{(0)}-E_{s^{\prime}}^{(0)}\right)\left(\mathrm{e}^{-\beta E_{s^{\prime}}^{(0)}}-\mathrm{e}^{-\beta E_{s}^{(0)}}\right) \\
& =\frac{1}{k^{2}} \cdot \frac{1}{Z^{(0)}} \operatorname{trace}\left\{\left[q_{l},\left[H^{(0)}, q_{l}\right]\right] \mathrm{e}^{-\beta H^{(0)}}\right\}=\frac{1}{m k^{2}},
\end{aligned}
$$

where $Z^{(0)}=\operatorname{trace} \exp \left(-\beta H^{(0)}\right)$ and [., .] stands for commutator. This yields in (5.41)

$$
\left\langle\left[\omega_{l}(\tau)-\omega_{l}^{(N)}(\tau)\right]^{2}\right\rangle_{\gamma_{\beta, \Lambda}} \leqslant \frac{2}{m \beta} \sum_{k \in \mathcal{K}_{N}^{c}} \frac{1}{k^{2}} \searrow 0, \quad N \rightarrow \infty .
$$




\section{Acknowledgements}

Yuri Kozitsky is grateful for kind hospitality extended to him at Bielefeld, Bochum, and Bonn Universities. His research was supported in part by the Polish Scientific Research Committee through the grant KBN 2 PO3A 02915 that is also acknowledged. Further financial support by the DFG through the SFB 343, the BiBoS Research Center and the EU-TMR Project ERB-FMRX-CT96-0075 is gratefully acknowledged by the authors.

\section{REFERENCES}

[1] Albeverio S., Høegh-Krohn R., Homogeneous random fields and quantum statistical mechanics, J. Funct. Anal. 19 (1975) 242-272.

[2] Albeverio S., Høegh-Krohn R., Mathematical Theory of Feynman Path Integrals, Lecture Notes in Math. 523, Springer, Berlin, 1976.

[3] Albeverio S., Kondratiev Yu.G., Kozitsky Yu.V., Absence of critical points for a class of quantum hierarchical models, Comm. Math. Phys. 187 (1997) 1-18.

[4] Albeverio S., Kondratiev Yu.G., Kozitsky Yu.V., Suppression of critical fluctuations by strong quantum effects in quantum lattice systems, Comm. Math. Phys. 194 (1998) 493512.

[5] Albeverio S., Kondratiev Yu.G., Kozitsky Yu.V., Röckner M., Euclidean Gibbs states of quantum lattice systems, in preparation.

[6] Albeverio S., Kondratiev Yu.G., Minlos R.A., Rebenko A.L., Small mass behaviour of quantum Gibbs states for lattice models with unbounded spins, J. Statist. Phys. 92 (1998) 1153-1172.

[7] Albeverio S., Kondratiev Yu.G., Röckner M., Tsikalenko T.V., Uniqueness of Gibbs states for quantum lattice systems, Probab. Theory Relat. Fields 108 (1997) 193-218.

[8] Albeverio S., Kondratiev Yu.G., Röckner M., Tsikalenko T.V., Uniqueness of Gibbs states on loop lattices, C. R. Acad. Sci. Paris, Sér. I 342 (1997) 1401-1406.

[9] Albeverio S., Kondratiev Yu.G., Röckner M., Tsikalenko T.V., Dobrushin's uniqueness for quantum lattice systems with nonlocal interaction, Comm. Math. Phys. 189 (1997) 621630.

[10] Albeverio S., Kondratiev Yu.G., Röckner M., Tsikalenko T.V., Glauber dynamics for quantum lattice systems, Rev. Math. Phys., to appear.

[11] Barbulyak V.S., Kondratiev Yu.G., Functional integrals and quantum lattice systems: I. Existence of Gibbs states, Reports Nat. Acad. Sci. of Ukraine 9 (1991) 38-40.

[12] Barbulyak V.S., Kondratiev Yu.G., Functional integrals and quantum lattice systems: II. Periodic Gibbs states, Reports Nat. Acad. Sci. of Ukraine 8 (1991) 31-34.

[13] Barbulyak V.S., Kondratiev Yu.G., Functional integrals and quantum lattice systems: III. Phase transitions, Reports Nat. Acad. Sci. of Ukraine 10 (1991) 19-21.

[14] Barbulyak V.S., Kondratiev Yu.G., A criterion for the existence of periodic Gibbs states of quantum lattice systems, Selecta Math. (N.S.) 12 (1993) 25-35.

[15] Berezin F.A., Shubin M.A., The Schrödinger Equation, Kluwer Academic Publishers, Dordrecht, 1991.

[16] Bratteli O., Robinson D.W., Operator Algebras and Quantum Statistical Mechanics, I, II, Springer, New York, 1981.

[17] Bruce A.D., Cowley R.A., Structural Phase Transition, Taylor and Francis, 1981. 
[18] Dobrushin R.L., Prescribing a system of random variables by conditional distributions, Theory Probab. Appl. 15 (1970) 458-486.

[19] Dobrushin R.L., Shlosman S.B., Constructive criterion for the uniqueness of Gibbs field, in: Statistical Physics and Dynamical Systems. Rigorous Results, Birkhäuser, Basel, 1985, pp. 347-370.

[20] Driesler W., Landau L., Perez J.F., Estimates of critical lengths and critical temperatures for classical and quantum lattice systems, J. Statist. Phys. 20 (1979) 123-162.

[21] Dyson F.J., Lieb E.H., Simon B., Phase transitions in quantum spin systems with isotropic and nonisotropic interactions, J. Statist. Phys. 18 (1978) 335-383.

[22] Georgii H.O., Gibbs Measures and Phase Transition, Walter de Gruyter, Springer, Berlin, 1988.

[23] Globa S.A., Kondratiev Yu.G., The construction of Gibbs states of quantum lattice systems, Selecta Math. Sov. 9 (1990) 297-307.

[24] Guerra F., Rosen L., Simon B., Boundary conditions in the $P(\varphi)_{2}$ Euclidean field theory, Ann. Inst. H. Poincaré 15 (1976) 231-334.

[25] Klein A., Landau L., Stochastic processes associated with KMS states, J. Funct. Anal. 42 (1981) 368-428.

[26] Kondratiev Yu.G., Phase transitions in quantum models of ferroelectrics, in: Stochastic Processes, Physics, and Geometry II, World Scientific, Singapore, 1994, pp. 465-475.

[27] Kozitsky Yu., Quantum effects in a lattice model of anharmonic vector oscillators, Letters in Math. Phys. (2000), to appear.

[28] Künsch H., Decay of correlations under Dobrushin's uniqueness condition and its applications, Comm. Math. Phys. 84 (1982) 207-222.

[29] Minlos R.A., Verbeure A., Zagrebnov V.A., A quantum crystal model in the light mass limit Gibbs state, Preprint-KUL-TF-97/16, 1997.

[30] Pastur L.A., Khoruzhenko B.A., Phase transitions in quantum models of rotators and ferroelectrics, Theoret. Math. Phys. 73 (1987) 111-124.

[31] Reed M., Simon B., Methods of Modern Mathematical Physics. Functional Analysis, Vol. I, Academic Press, New York, 1972.

[32] Schneider T., Beck H., Stoll E., Quantum effects in an $n$-component vector model for structural phase transition, Phys. Rev. B13 (1976) 1123-1130.

[33] Simon B., The $P(\varphi)_{2}$ Euclidean (Quantum) Field Theory, Princeton Univ. Press, 1974.

[34] Simon B., Functional Integrals in Quantum Physics, Academic Press, New York, 1986.

[35] Stamenković S., Unified model description of order-disorder and displacive structural phase transitions, Condensed Matter Physics 1(14) (1998) 257-309.

[36] Verbeure A., Zagrebnov V.A., No-Go theorem for quantum structural phase transition, J. Phys. A: Math. Gen. 28 (1995) 5415-5421. 